\title{
A Comparative Study of Dark Matter in the MSSM and Its Singlet Extensions: A Mini Review
}

\author{
Wenyu Wang \\ Institute of Theoretical Physics, College of Applied Science, Beijing University of Technology, \\ Beijing 100124, China \\ Correspondence should be addressed to Wenyu Wang, wywang@itp.ac.cn
}

Received 23 May 2012; Revised 28 July 2012; Accepted 12 September 2012

Academic Editor: Ulrich Ellwanger

Copyright (C) 2012 Wenyu Wang. This is an open access article distributed under the Creative Commons Attribution License, which permits unrestricted use, distribution, and reproduction in any medium, provided the original work is properly cited.

\begin{abstract}
In this note we briefly review the recent studies of dark matter in the MSSM and its singlet extensions: the NMSSM, the nMSSM, and the general singlet extension. Under the new detection results of CDMS II, XENON, CoGeNT, and PAMELA, we find that (i) the latest detection results can exclude a large part of the parameter space which is allowed by current collider constraints in these models. The future SuperCDMS and XENON can cover most of the allowed parameter space; (ii) the singlet sector will decouple from the MSSM-like sector in the NMSSM; however, singlet sector makes the nMSSM quite different from the MSSM; (iii) the NMSSM can allow light dark matter at several GeV to exist. Light CP-even or CP-odd Higgs boson must be present so as to satisfy the measured dark matter relic density. In case of the presence of a light CP-even Higgs boson, the light neutralino dark matter can explain the CoGeNT and DAMA/LIBRA results; (iv) the general singlet extension of the MSSM gives a perfect explanation for both the relic density and the PAMELA result through the Sommerfeld-enhanced annihilation. Higgs decays in different scenario are also studied.
\end{abstract}

\section{Introduction}

Although there are many theoretical or aesthetical arguments for the necessity of TeV-scale new physics, the most convincing evidence is from the (Wilkinson Microwave Anisotropy Probe) WMAP observation of the cosmic cold dark matter, which naturally indicates the existence of (weakly interacting massive particles) WIMPs beyond the prediction of the standard model (SM). By contrast, the neutrino oscillations may rather imply trivial new physics (plainly adding right-handed neutrinos to the SM) or new physics at some very high see-saw scale unaccessible to any foreseeable colliders. Therefore, the TeV-scale new physics to be unraveled at the large hadron collider (LHC) is the most likely related to the WIMP dark matter. 
If WIMP dark matter is chosen by nature, it will give a strong argument for low-energy supersymmetry (SUSY) with R parity which can give a good candidate. Nevertheless, SUSY is motivated for solving the hierarchy problem elegantly. It can also solve other puzzles of the $\mathrm{SM}$, such as the $3 \sigma$ deviation of the muon anomalous magnetic moment from the SM prediction. In the framework of SUSY, the most intensively studied model is the minimal supersymmetric standard model (MSSM) [1], which is the most economical realization of SUSY. However, this model suffers from the $\mu$-problem. The $\mu$-parameter is the only dimensional parameter in the SUSY conserving sector. From a top-down view, one would expect the $\mu$ to be either zero or at the Planck scale. But in the MSSM, the relation of the electroweak (EW) scale soft parameters $\left(\tilde{m}_{d}^{2}, \tilde{m}_{u}^{2}\right)[2]$

$$
\frac{1}{2} M_{Z}^{2}=\frac{\tilde{m}_{d}^{2}-\tilde{m}_{u}^{2} \tan ^{2} \beta}{\tan ^{2} \beta-1}-\mu^{2}
$$

makes that $\mu$ must be at the EW scale, while LEP constraints on the chargino mass require $\mu$ to be nonzero [3]. A simple solution is to promote $\mu$ to a dynamical field in extensions of the MSSM that contain an additional singlet superfield $\widehat{S}$ which does not interact with the MSSM fields other than the two Higgs doublets. An effective $\mu$ can be reasonably getten at EW scale when $\widehat{S}$ denotes the vacuum expectation value (VEV) of the singlet field. Among these extension models the next-to-minimal supersymmetric model (NMSSM) [4-27] and the nearly minimal supersymmetric model (nMSSM) [28-37] caused much attention recently. Note that the little hierarchy problem which is also a trouble of the MSSM is relaxed greatly in the NMSSM.

If we introduce a singlet superfield to the MSSM, the Higgs sector will have one more $\mathrm{CP}$ even component and one more $\mathrm{CP}$ odd component, and the neutralino sector will have one more singlino component. These singlet multiplets compose a "singlet sector" of the MSSM. It can make the phenomenologies of SUSY dark matter and Higgs different from the MSSM. More and more precision results of dark matter detection give us an opportunity to test if this singlet sector really exists. For example, experiments for the underground direct detection of cold dark matter $\tilde{X}$ have recently made significant progress while the null observation of $\tilde{X}$ in the CDMS and XENON100 experiments has set rather tight upper limits on the spin-independent (SI) cross-section of $\tilde{\chi}$-nucleon scattering [38-40]. The CoGeNT experiment [41] reported an excess which cannot be explained by any known background sources but seems to be consistent with the signal of a light $\tilde{x}$ with mass around $10 \mathrm{GeV}$ and scattering rate $(1-2) \times 10^{-40} \mathrm{~cm}^{2}$. Intriguingly, this range of mass and scattering rate is compatible with dark matter explanation for both the DAMA/LIBRA data and the preliminary CRESST data [42]. Though CoGeNT result is not consistent with the CDMS or XENON results, it implies that the mass of dark matter can range a very long scope at EW scale, that is, from a few $\mathrm{GeV}$ to several $\mathrm{TeV}$. The indirect detection PAMELA also observed an excess of the cosmic ray positron in the energy range $10-100 \mathrm{GeV}[43,44]$ which may be explained by dark matter.

In this paper, we will give a short review on the difference between the MSSM and the MSSM with a singlet sector under the constraints of new dark matter detection results. As the Higgs hunting on colliders has delicate relation with dark matter detections, the implication on Higgs searching is also reviewed. The content is based on our previous work [45-47]. The paper is organized as follows: in Section 2, we will give a short review on the structures of the MSSM, the NMSSM, and the nMSSM. In Section 3 we will give a comparison on the 
models under the constraints of CDMS, XENON, and CoGeNT. In Section 4, a general singlet extension of the MSSM is discussed, and a summary is given in Section 5.

\section{The MSSM and Its Singlet Extensions}

As an economical realization of supersymmetry, the MSSM has the minimal content of particles, while the NMSSM and the nMSSM extend the MSSM by only adding one singlet Higgs superfield $\widehat{S}$. The difference between these models is reflected in their superpotential:

$$
\begin{gathered}
W_{\mathrm{MSSM}}=W_{F}+\mu \widehat{H}_{u} \cdot \widehat{H}_{d}, \\
W_{\mathrm{NMSSM}}=W_{F}+\lambda \widehat{H}_{u} \cdot \widehat{H}_{d} \widehat{S}+\frac{1}{3} \kappa \widehat{S}^{3}, \\
W_{\mathrm{nMSSM}}=W_{F}+\lambda \widehat{H}_{u} \cdot \widehat{H}_{d} \widehat{S}+\xi_{F} M_{n}^{2} \widehat{S},
\end{gathered}
$$

where $W_{F}=Y_{u} \widehat{Q} \cdot \widehat{H}_{u} \widehat{U}-Y_{d} \widehat{Q} \cdot \widehat{H}_{d} \widehat{D}-Y_{e} \widehat{L} \cdot \widehat{H}_{d} \widehat{E}$ with $\widehat{Q}, \widehat{U}$, and $\widehat{D}$ being the squark superfields, and $\widehat{L}$ and $\widehat{E}$ being the slepton superfields. $\widehat{H}_{u}$ and $\widehat{H}_{d}$ are the Higgs doublet superfields, $\lambda, \kappa$, and $\xi_{F}$ are dimensionless coefficients, and $\mu$ and $M_{n}$ are parameters with mass dimension. Note that there is no explicit $\mu$-term in the NMSSM or the nMSSM, and an effective $\mu$-parameter (denoted as $\mu_{\text {eff }}$ ) can be generated when the scalar component $(S)$ of $\widehat{S}$ develops a VEV. Also note that the nMSSM differs from the NMSSM in the last term with the trilinear singlet term $\kappa \widehat{S}^{3}$ of the NMSSM replaced by the tadpole term $\xi_{F} M_{n}^{2} \widehat{S}$. As pointed out in [28-36], such a tadpole term can be generated at a high loop level and naturally be of the SUSY breaking scale. The advantage of such replacement is the nMSSM that has no discrete symmetry thus free of the domain wall problem which the NMSSM suffers from.

Corresponding to the superpotential, the Higgs soft terms in the scalar potentials are also different between the three models (the soft terms for gauginos and sfermions are the same thus not listed here)

$$
\begin{gathered}
V_{\mathrm{soft}}^{\mathrm{MSSM}}=\tilde{m}_{d}^{2}\left|H_{d}\right|^{2}+\tilde{m}_{u}^{2}\left|H_{u}\right|^{2}+\left(B \mu H_{u} \cdot H_{d}+\text { h.c. }\right) \\
V_{\mathrm{soft}}^{\mathrm{NMSSM}}=\tilde{m}_{d}^{2}\left|H_{d}\right|^{2}+\tilde{m}_{u}^{2}\left|H_{u}\right|^{2}+\tilde{m}_{s}^{2}|S|^{2}+\left(A_{\curlywedge} \lambda S H_{d} \cdot H_{u}+\frac{\kappa}{3} A_{\kappa} S^{3}+\text { h.c. }\right) \\
V_{\mathrm{soft}}^{\mathrm{nMSSM}}=\tilde{m}_{d}^{2}\left|H_{d}\right|^{2}+\tilde{m}_{u}^{2}\left|H_{u}\right|^{2}+\tilde{m}_{s}^{2}|S|^{2}+\left(A_{\curlywedge} \lambda S H_{d} \cdot H_{u}+\xi_{S} M_{n}^{3} S+\text { h.c. }\right) .
\end{gathered}
$$

After the scalar fields $H_{u}, H_{d}$, and $S$ develop their VEVs $v_{u}, v_{d}$, and $s$, respectively, they can be expanded as

$$
H_{d}=\left(\begin{array}{c}
\frac{1}{\sqrt{2}}\left(v_{d}+\phi_{d}+i \varphi_{d}\right) \\
H_{d}^{-}
\end{array}\right), \quad H_{u}=\left(\begin{array}{c}
H_{u}^{+} \\
\frac{1}{\sqrt{2}}\left(v_{u}+\phi_{u}+i \varphi_{u}\right)
\end{array}\right), \quad S=\frac{1}{\sqrt{2}}(s+\sigma+i \xi)
$$


The mass eigenstates can be obtained by unitary rotations

$$
\left(\begin{array}{c}
h_{1} \\
h_{2} \\
h_{3}
\end{array}\right)=U^{H}\left(\begin{array}{c}
\phi_{d} \\
\phi_{u} \\
\sigma
\end{array}\right), \quad\left(\begin{array}{c}
a_{1} \\
a_{2} \\
G_{0}
\end{array}\right)=U^{A}\left(\begin{array}{c}
\varphi_{d} \\
\varphi_{u} \\
\xi
\end{array}\right), \quad\left(\begin{array}{c}
G^{+} \\
H^{+}
\end{array}\right)=U^{H^{+}}\left(\begin{array}{c}
H_{d}^{+} \\
H_{u}^{+}
\end{array}\right),
$$

where $h_{1,2,3}$ and $a_{1,2}$ are, respectively, the CP-even and CP-odd neutral Higgs bosons, $G^{0}$ and $\mathrm{G}^{+}$are Goldstone bosons, and $\mathrm{H}^{+}$is the charged Higgs boson. Including the scalar part of the singlet sector in the NMSSM and the nMSSM leads to a pair of charged Higgs bosons, three $\mathrm{CP}$-even and two CP-odd neutral Higgs bosons. In the MSSM, we only have two CP-even and one $\mathrm{CP}$-odd neutral Higgs bosons in addition to a pair of charged Higgs bosons.

The MSSM predicts four neutralinos $x_{i}^{0}(i=1,2,3,4)$, that is, the mixture of neutral gauginos (bino $\lambda^{\prime}$ and neutral wino $\left.\lambda^{3}\right)$ and neutral higgsinos $\left(\psi_{H_{u^{\prime}}}^{0} \psi_{H_{d}}^{0}\right)$, while the NMSSM and the nMSSM predict one more neutralino corresponding to the singlino $\psi_{S}$ from the fermion part of singlet sector. In the basis $\left(-i \lambda^{\prime},-i \lambda^{3} 2, \psi_{H_{u^{\prime}}}^{0} \psi_{H_{d^{\prime}}}^{0} \psi_{S}\right.$ ) (for the MSSM $\psi_{S}$ is absent) the neutralino mass matrix is given by

$$
\left(\begin{array}{ccccc}
M_{1} & 0 & m_{Z} s_{W} s_{b} & -m_{Z} s_{W} c_{b} & 0 \\
0 & M_{2} & -m_{Z} c_{W} s_{b} & m_{Z} c_{W} c_{b} & 0 \\
m_{Z} s_{W} s_{b} & -m_{Z} s_{W} s_{b} & 0 & -\mu & -\lambda v c_{b} \\
-m_{Z} s_{W} c_{b} & -m_{Z} c_{W} c_{b} & -\mu & 0 & -\lambda v s_{b} \\
0 & 0 & -\lambda v c_{b} & -\lambda v s_{b}
\end{array} \quad\left\{\begin{array}{l}
2 \frac{\kappa}{\lambda} \mu \text { for the NMSSM } \\
0 \text { for the nMSSM }
\end{array}\right),\right.
$$

where $M_{1}$ and $M_{2}$ are, respectively, $U(1)$ and $S U(2)$ soft gaugino mass parameters, $s_{W}=$ $\sin \theta_{W}, c_{W}=\cos \theta_{W}, s_{b}=\sin \beta$, and $c_{b}=\cos \beta$ with $\tan \beta \equiv v_{u} / v_{d}$. The lightest neutralino $\tilde{\chi}_{1}^{0}$ is assumed to be the lightest supersymmetric particle (LSP), serving as the SUSY dark matter particle. It is composed by

$$
\tilde{X}_{1}^{0}=N_{11}\left(-i \lambda^{\prime}\right)+N_{12}\left(-i \lambda^{3}\right)+N_{13} \psi_{H_{u}}^{0}+N_{14} \psi_{H_{d}}^{0}+N_{15} \psi_{S},
$$

where $N$ is the unitary matrix $\left(N_{15}\right.$ is zero for the MSSM) to diagonalize the mass matrix in (2.7). For the mass matrices above we should note the following two points.

(1) For a moderate value of $\kappa$, the neutralino sector of the NMSSM can go back to the MSSM when $\lambda$ approaches to zero. This is because in such case the singlino component will become super heavy and decouple from EW scale. The singlet scalar will not mix with the two Higgs doublet, and then the NMSSM will be almost the same as the MSSM at EW scale.

(2) Since the $\psi_{S} \psi_{S}$ element of (2.7) is zero in the nMSSM, the singlino will not decouple when $\lambda$ approaches to zero. In fact, in the nMSSM the mass of the LSP can be written as

$$
m_{\chi_{1}^{0}} \simeq \frac{2 \mu_{\mathrm{eff}} \lambda^{2}\left(v_{u}^{2}+v_{d}^{2}\right)}{2 \mu_{\mathrm{eff}}^{2}+\lambda^{2}\left(v_{u}^{2}+v_{d}^{2}\right)} \frac{\tan \beta}{\tan ^{2} \beta+1}
$$

This formula shows that, to get a heavy $\tilde{X}_{1}^{0}$, we need a large $\lambda$, a small $\tan \beta$ as well as a moderate $\mu_{\text {eff. }}$ 
The chargino sector of these three models is the same except that in the NMSSM/ nMSSM the parameter $\mu$ is replaced by $\mu_{\text {eff. }}$. The charginos $\tilde{X}_{1,2}^{ \pm}\left(m_{X_{1}^{ \pm}} \leq m_{X_{2}^{ \pm}}\right)$are the mixture of charged Higgsinos $\psi_{H_{u, d}}^{ \pm}$and winos $\lambda^{ \pm}=\left(\lambda^{1} \pm \lambda^{2}\right) / \sqrt{2}$, whose mass matrix in the basis of $\left(-i \lambda^{ \pm}, \psi_{H_{u, d}}^{ \pm}\right)$is given by

$$
\left(\begin{array}{cc}
M_{2} & \sqrt{2} m_{W} s_{b} \\
\sqrt{2} m_{W} c_{b} & \mu_{\mathrm{eff}}
\end{array}\right)
$$

So the chargino $\widetilde{X}_{1}^{ \pm}$can be wino dominant (when $M_{2}$ is much smaller than $\mu$ ) or higgsino dominant (when $\mu$ is much smaller than $M_{2}$ ). Since the composing property (wino-like, binolike, higgsino-like, or singlinolike) of the LSP and the chargino $\tilde{X}_{1}^{ \pm}$is very important in SUSY phenomenologies, we will show such a property in our following study.

\section{Comparison with the MSSM and the MSSM with a Singlet Sector}

\subsection{In Light of CDMS II and XENON}

First let us see the MSSM, the NMSSM, and the nMSSM under the constraints of results of CDMS II and XENON100. As both current and future limits of $\tilde{X}$-nucleon of CDMS and XENON are similar to each other, we will show only one of them. Nevertheless, as a good substitute of the SM, SUSY model must satisfy all the results of current collider and detector measurements. In our study we consider the following experimental constraints [48]: (1) we require $\tilde{X}_{1}^{0}$ to account for dark matter relic density $0.105<\Omega h^{2}<0.119$; (2) we require the SUSY contribution to explain the deviation of the muon $a_{\mu}$, that is, $a_{\mu}^{\exp }-a_{\mu}^{\mathrm{SM}}=(25.5 \pm 8.0) \times 10^{-10}$, at $2 \sigma$ level; (3) the LEP-I bound on the invisible Z-decay, $\Gamma\left(Z \rightarrow \widetilde{X}_{1}^{0} \widetilde{X}_{1}^{0}\right)<1.76 \mathrm{MeV}$, and the LEP-II upper bound on $\sigma\left(e^{+} e^{-} \rightarrow \tilde{X}_{1}^{0} \tilde{X}_{i}^{0}\right)$, which is $5 \times 10^{-2} \mathrm{pb}$ for $i>1$, as well as the lower mass bounds on sparticles from direct searches at LEP and the Tevatron; (4) the constraints from the direct search for Higgs bosons at LEPII, including the decay modes $h \rightarrow h_{1} h_{1}, a_{1} a_{1} \rightarrow 4 f$, which limit all possible channels for the production of the Higgs bosons; (5) the constraints from $B$ physics observable such as $B \rightarrow X_{s} \gamma, B_{s} \rightarrow \mu^{+} \mu^{-}, B^{+} \rightarrow \tau^{+} v, \Upsilon \rightarrow \gamma a_{1}$, the $a_{1}-\eta_{b}$ mixing, and the mass difference $\Delta M_{d}$ and $\Delta M_{s} ;(6)$ the constraints from the precision EW observable such as $\rho_{\text {lept }}, \sin ^{2} \theta_{\text {eff }}^{\text {lept }}$, $m_{W}$, and $R_{b} ;(7)$ the constraints from the decay $\Upsilon \rightarrow \gamma h_{1}$, and the Tevatron search for a light Higgs boson via $4 \mu$ and $2 \mu 2 \tau$ signals [49]. The constraints (1.1)-(2.2) have been encoded in the package NMSSMTools [50]. We use this package in our calculation and extend it by adding the constraints (2.3), (2.4). As pointed out in [49], the constraints (2.4) are important for a light Higgs boson. In addition to the above experimental limits, we also consider the constraint from the stability of the Higgs potential, which requires that the physical vacuum of the Higgs potential with nonvanishing VEVs of Higgs scalars should be lower than any local minima.

For the calculation of cross-section of $\tilde{X}$-nucleon scattering, we use the formulas in $[51,52]$ for the MSSM and extend them to the NMSSM/nMSSM. It is sufficient to consider only the SI interactions between $\tilde{X}_{1}^{0}$ and nucleon (denoted by $f_{p}$ for proton and $f_{n}$ for neutron [52]) in the calculation. The leading order of these interactions is induced by exchanging the 
SM-like Higgs boson at tree level. For moderately light Higgs bosons, $f_{p}$ is approximated by [52] (similarly for $f_{n}$ )

$$
f_{p} \simeq \sum_{q=u, d, s} \frac{f_{q}^{H}}{m_{q}} m_{p} f_{T_{q}}^{(p)}+\frac{2}{27} f_{T_{G}} \sum_{q=c, b, t} \frac{f_{q}^{H}}{m_{q}} m_{p}
$$

where $f_{T q}^{(p)}$ denotes the fraction of $m_{p}$ (proton mass) from the light quark $q$ while $f_{T_{G}}=1$ $\sum_{u, d, s} f_{T_{q}}^{(p)}$ is the heavy quark contribution through gluon exchange. $f_{q}^{H}$ is the coefficient of the effective scalar operator. The $\tilde{x}^{0}$-nucleus scattering rate is then given by [52]

$$
\sigma^{\mathrm{SI}}=\frac{4}{\pi}\left(\frac{m_{\tilde{\chi}^{0}} m_{T}}{m_{\tilde{\chi}^{0}}+m_{T}}\right)^{2} \times\left(n_{p} f_{p}+n_{n} f_{n}\right)^{2},
$$

where $m_{T}$ is the mass of target nucleus, and $n_{p}\left(n_{n}\right)$ is the number of proton (neutron) in the target nucleus. In our numerical calculations we take $f_{T_{u}}^{(n)}=0.023, f_{T_{d}}^{(n)}=0.034, f_{T_{u}}^{(p)}=0.019$, $f_{T_{d}}^{(p)}=0.041$, and $f_{T_{s}}^{(p)}=f_{T_{s}}^{(n)}=0.38$. Note that the scattering rate is very sensitive to the value of $f_{T_{s}}[53,54]$. Recent lattice simulation [55-57] gave a much smaller value of $f_{T_{s}}(0.020)$, and it reduces the scattering rate significantly which can be seen in $[58,59]$.

Considering all the constraints listed above, we scan over the parameters in the following ranges:

$$
\begin{gathered}
100 \mathrm{GeV} \leq\left(M_{\tilde{q}}, M_{\tilde{\ell}}, m_{A}, \mu\right) \leq 1 \mathrm{TeV}, \\
50 \mathrm{GeV} \leq M_{1} \leq 1 \mathrm{TeV}, \quad 1 \leq \tan \beta \leq 40, \\
(|\lambda|,|\kappa|) \leq 0.7, \quad\left|A_{\kappa}\right| \leq 1 \mathrm{TeV},
\end{gathered}
$$

where $M_{\tilde{q}}$ and $M_{\tilde{\ell}}$ are the universal soft mass parameters of the first two generations of squarks and the three generations of sleptons, respectively. To reduce the number of the relevant soft parameters, we worked in the so-called $m_{h}^{\max }$ scenario with the following choice of the soft masses for the third generation squarks: $M_{\tilde{Q}_{3}}=M_{\tilde{U}_{3}}=M_{\tilde{D}_{3}}=800 \mathrm{GeV}$, and $X_{t}=$ $A_{t}-\mu \cot \beta=-1600 \mathrm{GeV}$. The advantage of such a choice is that other SUSY parameters more easily survive the constraints (so that the bounds we obtain are conservative). Moreover, we assume the grand unification relation for the gaugino masses:

$$
M_{1}: M_{2}: M_{3} \simeq 1: 1.83: 5.26
$$

This relation is often assumed in studies of SUSY at the TeV scale for it can be easily generated in the mSUGRA model [60]. Note that relaxing this relation will give a large effect on the light neutralino scenario [61-64].

The surviving points for the three model are displayed in Figure 1 for the spinindependent elastic cross-section of $\tilde{X}$-nucleon scattering. We see that for each model the CDMS II limits can exclude a large part of the parameter space allowed by current collider constraints, and the future SuperCDMS $(25 \mathrm{~kg})$ limits can cover most of the allowed 


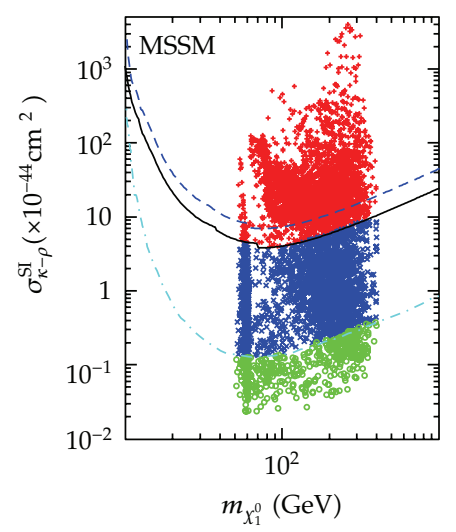

(a)

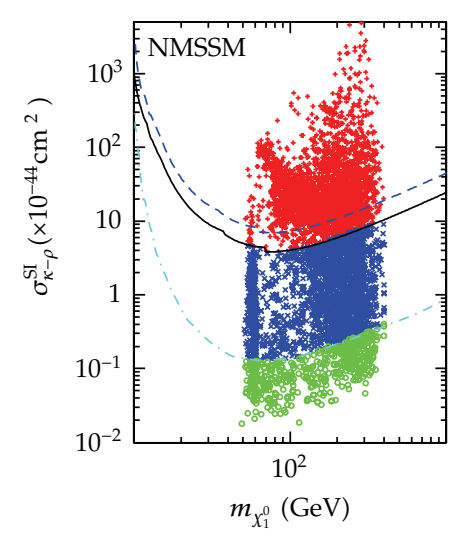

(b)

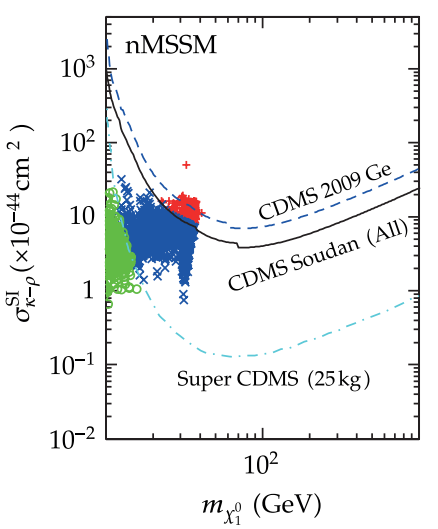

(c)

Figure 1: The scatter plots (taken for [46]) for the spin-independent elastic cross-section of $\tilde{X}^{\text {-nucleon }}$ scattering. The "+" points (red) are excluded by CDMS limits (solid line), and the " $\times$ " (blue) would be further excluded by SuperCDMS $25 \mathrm{~kg}$ [65] in case of nonobservation (dash-dotted line), and the "o" (green) are beyond the SuperCDMS sensitivity.

parameter space. For the MSSM and the NMSSM dark matter mass is roughly in range of $50-400 \mathrm{GeV}$, while for the nMSSM dark matter mass is constrained below $40 \mathrm{GeV}$ by current experiments and further constrained below $20 \mathrm{GeV}$ by SuperCDMS in case of nonobservation.

From Figure 1, we can see that the $\tilde{x}$-nucleon scattering plot of the MSSM and the NMSSM is very similar to each other, but very different from nMSSM. This implies that, under the experiment constraints, the singlet sector will decouple from the MSSM-like sector in the NMSSM, and then the NMSSM will perform almost the same as the MSSM. However, the singlet components change EW scale phenomenology greatly in the nMSSM. This can also be seen in Figures 2 and 3. We can see that for both the MSSM and the NMSSM $\tilde{X}_{1}^{0}$ is bino dominant, while for the nMSSM $\tilde{\chi}_{1}^{0}$ is singlino-dominant, and the region allowed by CDMS limits (and SuperCDMS limits in case of nonobservation) favors a more bino-like $\widetilde{\chi}_{1}^{0}$ for the MSSM/NMSSM and a more singlinolike $\tilde{x}_{1}^{0}$ for the nMSSM. For the MSSM/NMSSM the LSP lower bound around $50 \mathrm{GeV}$ is from the chargino lower bound of $103.5 \mathrm{GeV}$ plus the assumed GUT relation $M_{1} \simeq 0.5 M_{2}$, while the upper bound around $400 \mathrm{GeV}$ is from the binonature of the LSP ( $M_{1}$ cannot be too large and must be much smaller than other relevant parameters) plus the experimental constraints like the muon g-2 and B-physics. If we do not assume the GUT relation $M_{1} \simeq 0.5 M_{2}$, then $M_{1}$ can be as small as $40 \mathrm{GeV}$, and the LSP lower bound in the MSSM/NMSSM will not be sharply at $50 \mathrm{GeV}$. (We will talk about it in the following section.) For both the MSSM and the NMSSM, the CDMS limits tend to favor a heavier chargino and ultimately the SuperCDMS limits tend to favor a wino-dominant chargino with mass about $2 m_{x_{1}^{0}}$. Note that there still can be a singlino-dominant LSP in some parameter space of the NMSSM [66], but, in the scan range (3.3) listed above, getting such singlino-dominant LSP needs some fine-tuning, thus we do not focus on it.

In Figure 4 we show the value of $|\lambda|$ versus the charged Higgs mass in the NMSSM and the nMSSM. This figure indicates that $\lambda$ larger than 0.4 is disfavored by the NMSSM. The underlying reason is that $h_{1} \widetilde{X}_{1}^{0} \tilde{X}_{1}^{0}$ depends on $\lambda$ explicitly, and large $\lambda$ can enhance $\tilde{X}$-nucleon scattering rate. By contrast, although CDMS has excluded some points with large $\lambda$ in the nMSSM, there are still many surviving points with $\lambda$ as large as 0.7 . We have talked about the reason above to get a heavy $x_{1}^{0}$, one needs a large $\lambda$, a small $\tan \beta$ as well as a moderate $\mu_{\text {eff. }}$. 


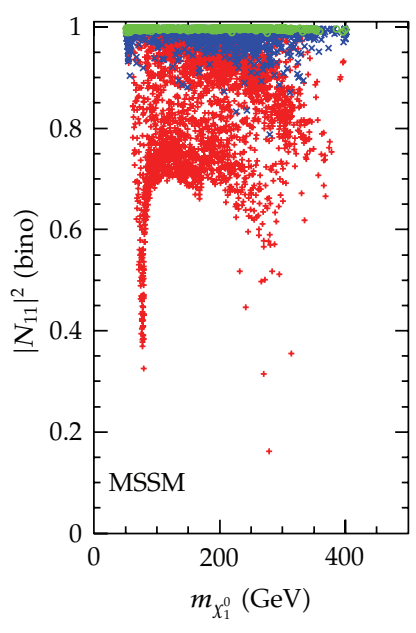

(a)

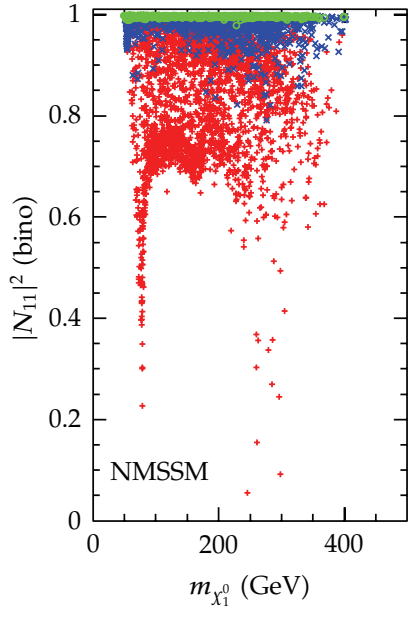

(b)

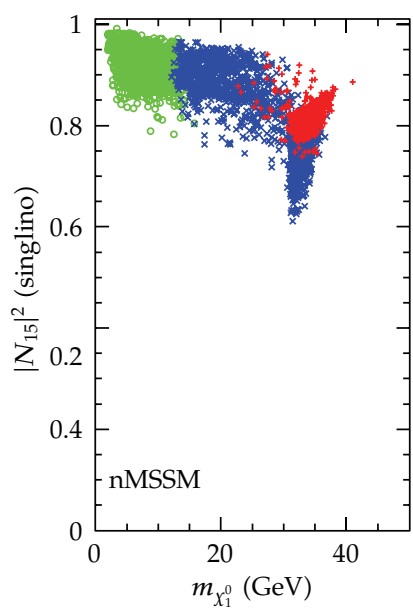

(c)

Figure 2: Same as Figure 1, but projected on the plane of $\left|N_{11}\right|^{2}$ and $\left|N_{15}\right|^{2}$ versus dark matter mass (taken for [46]).

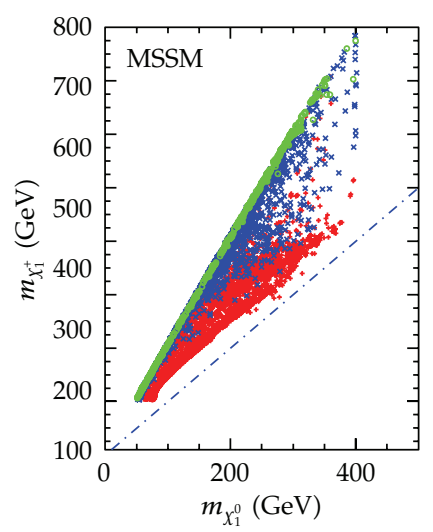

(a)

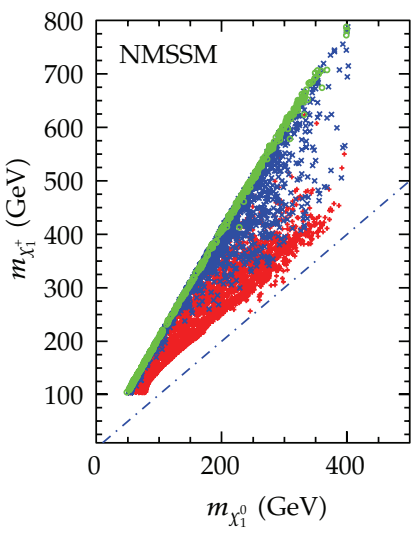

(b)

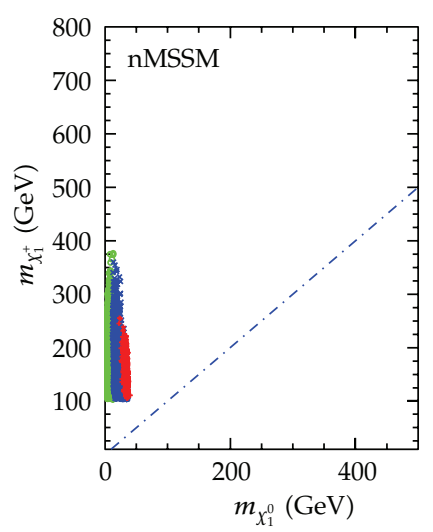

(c)

Figure 3: Same as Figure 1, but showing the chargino mass $m_{X_{1}^{+}}$versus the LSP mass. The dashed lines indicate $m_{X_{1}^{+}}=m_{X_{1}^{0}}($ taken for [46]).

From the survived parameter space for all the model above, we should know that the Higgs decay will be similar for the MSSM and the NMSSM, but quite different from the nMSSM. This can be seen in Figure 5 which shows decay branching ratio of $h_{1} \rightarrow \tilde{x}_{1}^{0} \tilde{\chi}_{1}^{0}$ versus the mass of the SM-like Higgs boson $h_{\mathrm{SM}}$ (which is $h_{1}$ here, and it is Higgs doublet $\widehat{H}_{u}$ and $\widehat{H}_{d}$ dominant). Such a decay is strongly correlated to the $\tilde{\chi}$-nucleon scattering because the coupling $h_{1} \tilde{\chi}_{1}^{0} \tilde{\chi}_{1}^{0}$ is involved in both processes. We see that in the MSSM and the NMSSM this decay mode can open only in a very narrow parameter space since $\tilde{\chi}_{1}^{0}$ cannot be so light, and in the allowed region this decay has a very small branching ratio (below $10 \%$ ). However, in the nMSSM this decay can open in a large part of the parameter space since the LSP can be very light, and its branching ratio can be quite large (over $80 \%$ or $90 \%$ ). 


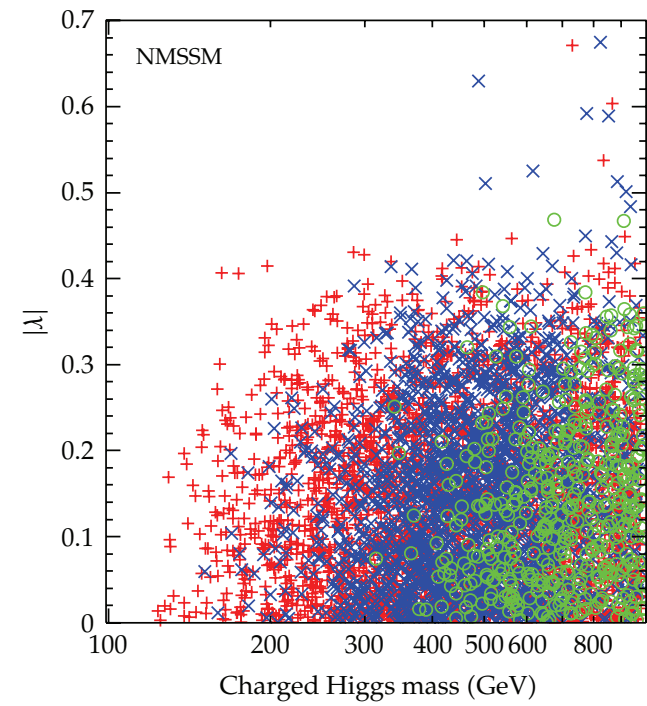

(a)

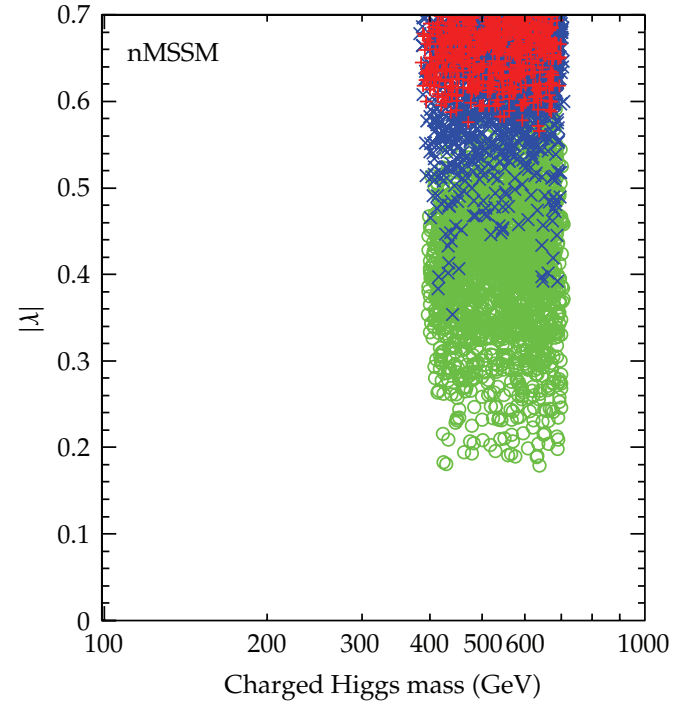

(b)

Figure 4: Same as Figure 1, but projected on the plane of $|\lambda|$ versus the charged Higgs mass in the NMSSM and the nMSSM (taken for [46]).

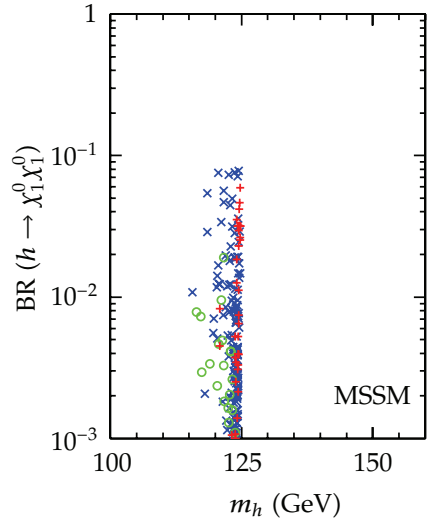

(a)

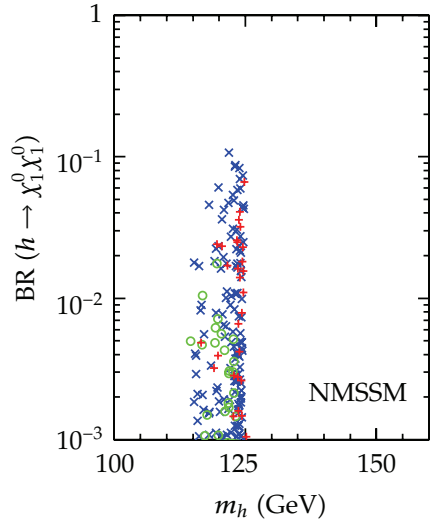

(b)

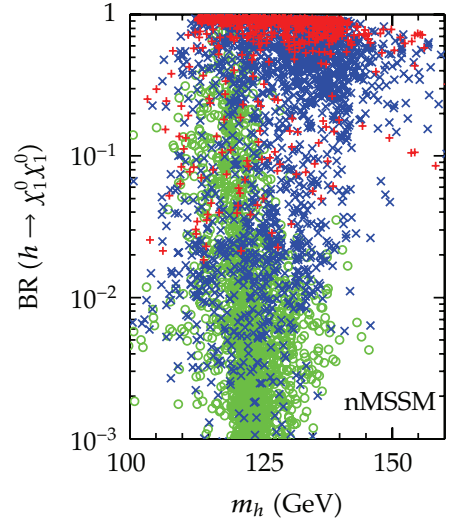

(c)

Figure 5: Same as Figure 1, but projected for the decay branching ratio of $h_{\mathrm{SM}} \rightarrow x_{1}^{0} x_{1}^{0}$ versus the mass of the Higgs boson $h_{\mathrm{SM}}$ (taken for [46]).

\subsection{Light Dark Matter in the NMSSM}

As we talked in the introduction, the data of CoGeNT experiment favors a light dark matter around $10 \mathrm{GeV}$. However, we scan the parameter space in the MSSM and find that it is very difficult to find a neutralino $\tilde{X}_{1}^{0}$ lighter than about $28 \mathrm{GeV}$, unless when it is associated with a light stau as the next to the lightest supersymmetric particle (NLSP), but such scenario always needs a fine tuning in the parameter space [67-70]. The main reason for the absence of a lighter $\widetilde{X}_{1}^{0}$ is that the dominant annihilation channel for $\tilde{X}_{1}^{0}$ in the early universe is $\tilde{X}_{1}^{0} \widetilde{X}_{1}^{0} \rightarrow b \bar{b}$ through s-channel exchange of the pseudoscalar Higgs boson $(A)$, and the measured dark 
matter relic density requires $m_{A} \sim(90-100) \mathrm{GeV}$ and $\tan \beta \sim 50$ and this is in conflict with the constraints from the LEP experiment and $B$ physics [71-74]. The LHC data gives an even more stronger constraint on the light pseudoscalar scenario $[75,76]$ such that light dark matter seems impossible in the MSSM. Though in the nMSSM the neutralino $\tilde{X}_{1}^{0}$ can be as light as $10 \mathrm{GeV}$ (shown in Figure 1), the scattering rate is much lower under the CoGeNTfavored region. In the NMSSM, however, with the participation of singlet sector one can get very light [4-27] Higgs. This feature is particularly useful for light $\tilde{\chi}_{1}^{0}$ scenario since it opens up new important annihilation channels for $\widetilde{x}_{1}^{0}$, that is, either into a pair of $h_{1}$ (or $a_{1}$ ) or into a pair of fermions via s-channel exchange of $h_{1}\left(\right.$ or $\left.a_{1}\right)$ [74, 77-79]. For the former case, $\tilde{x}_{1}^{0}$ must be heavier than $h_{1}\left(a_{1}\right)$, while, for the latter case, due to the very weak couplings of $h_{1}\left(a_{1}\right)$ with $\widetilde{X}_{1}^{0}$ and with the SM fermions, a resonance enhancement (i.e., $m_{h_{1}}$ or $m_{a_{1}}$ must be close to $\left.2 m_{\tilde{X}_{1}^{0}}\right)$ is needed to accelerate the annihilation. So a light $\tilde{X}_{1}^{0}$ may be necessarily accompanied by a light $h_{1}$ or $a_{1}$ to provide the required dark matter relic density. From the discussion in the upper section, light $\tilde{X}_{1}^{0}$ can be obtained by releasing the GUT relation (3.4); thus LSP in the NMSSM may explain the detection of CoGeNT. Note that as the LSP in the nMSSM is singlino dominant, relaxing the GUT relation will not the change the phenomenology of dark matter and Higgs too much.

Now we discuss how to get a light $h_{1}$ or $a_{1}$ in the NMSSM. A light $a_{1}$ can be easily obtained when the theory is close to the $U(1)_{R}$ or $U(1)_{\mathrm{PQ}}$ symmetry limit, which can be realized by setting the product $\kappa A_{\kappa}$ to be negatively small [4-27]. In contrast, a light $h_{1}$ cannot be obtained easily. However, as shown below, it can still be achieved by somewhat subtle cancelation via tuning the value of $A_{\kappa}$. We note that, for any theory with multiple Higgs fields, the existence of a massless Higgs boson implies the vanishing of the determinant of its squared mass matrix and vice versa. For the NMSSM, at tree level the parameter $A_{\kappa}$ only enters the mass term of the singlet Higgs bosons, so the determinant (Det $\mathcal{M}^{2}$ ) of the mass matrix of the CP-even Higgs bosons depends on $A_{\kappa}$ linearly [4-27]. When other relevant parameters are fixed, one can then obtain a light $h_{1}$ by varying $A_{\mathcal{K}}$ around the value $\tilde{A}_{\kappa}$ which is the solution to the equation Det $\mathcal{M}^{2}=0$. In practice, one must include the important radiative corrections to the Higgs mass matrix, which will complicate the dependence of $\boldsymbol{M}^{2}$ on $A_{\kappa}$. However, we checked that the linear dependence is approximately maintained by choosing the other relevant parameters at the SUSY scale, and one can solve the equation iteratively to get the solution $\widetilde{A}_{\kappa}$.

In Figure 6 we display the surviving parameter samples, showing the $\tilde{X}$-nucleon scattering cross-section versus the neutralino dark matter mass (left frame) and versus the mass of $h_{1}$ or $a_{1}$ (right frame). It shows that the scattering rate of the light dark matter can reach the sensitivity of CDMS, and, consequently, a sizable parameter space is excluded by the CDMS data [65]. The future CDMS experiment can further explore (but cannot completely cover) the remained parameter space. Note that in the light- $h_{1}$ case the scattering rate can be large enough to reach the sensitivity of CoGeNT and to cover the CoGeNT-favored region. The underlying reason is that the $\tilde{x}$-nucleon scattering can proceed through the $t$ channel exchange of the CP-even Higgs bosons, which can be enhanced by a factor $1 / m_{h_{1}}^{4}$ for a light $h_{1}[77,78]$, while a light $a_{1}$ can not give such an enhancement because the CP-odd Higgs bosons do not contribute to the scattering in this way. We noticed that the studies in $[73,80]$ claimed that the NMSSM is unable to explain the CoGeNT data because they did not consider the light- $h_{1}$ case.

In the light $\tilde{X}_{1}^{0}$ scenario, $h_{\mathrm{SM}}$ may decay exotically into $\tilde{X}_{i}^{0} \tilde{X}_{j}^{0}, h_{1} h_{1}$ or $a_{1} a_{1}$, and consequently the conventional decays are reduced. This feature is illustrated in Figure 7, which 


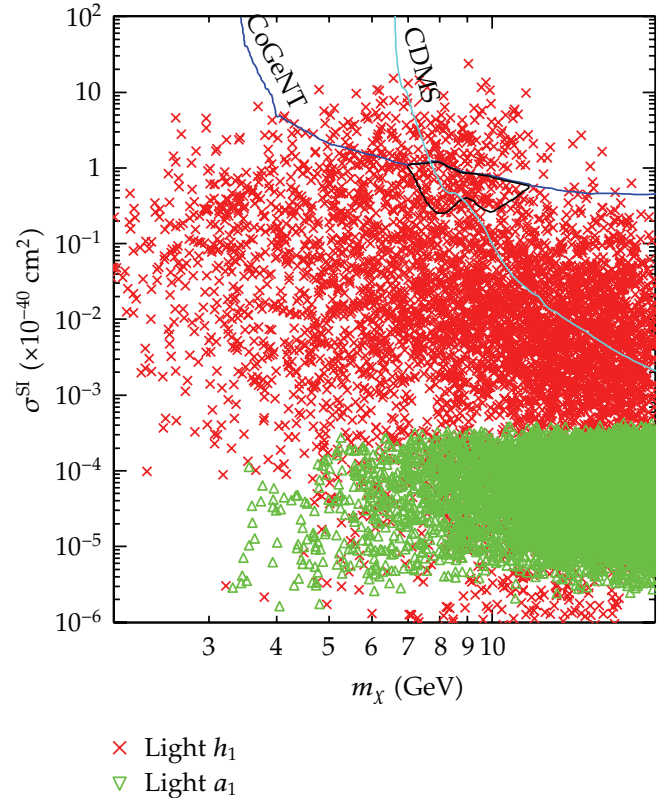

(a)

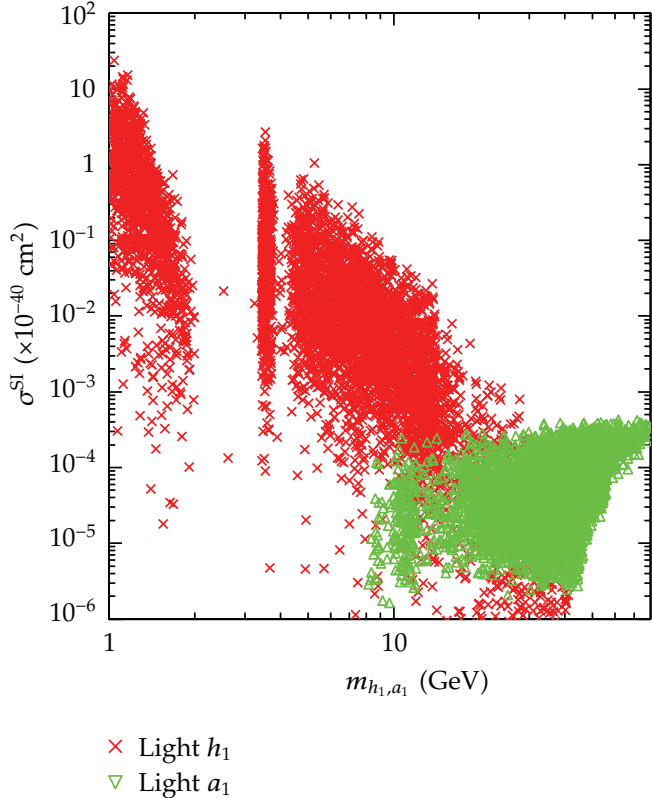

(b)

Figure 6: The scatter plots (taken for [47]) of the parameter samples which survive all constraints, with " $x$ " (red) and "ה" (green) corresponding to a light $h_{1}$ and a light $a_{1}$, respectively. The left frame is projected on the $\sigma^{\mathrm{SI}}-m_{X}$ plane, while the right frame is projected on the $\sigma^{\mathrm{SI}}-m_{h_{1}}$ plane (denoted by " $\times^{\prime \prime}$ ) and the $\sigma^{\mathrm{SI}}-m_{a_{1}}$ plane (denoted by " $\boldsymbol{\Delta}^{\prime \prime}$ ). The curves are the limits from CoGeNT [41] and CDMS [38], while the contour is the CoGeNT-favored region [41].

shows that the sum of the exotic decay branching ratios may exceed $50 \%$ and the traditional decays $h_{\mathrm{SM}} \rightarrow b \bar{b}, \tau \bar{\tau}, W W^{*}, \gamma \gamma$ can be severely suppressed. Numerically, we find that the branching ratio of $h_{\mathrm{SM}} \rightarrow b \bar{b}$ is suppressed to be below $30 \%$ for all the surviving samples in the light- $h_{1}\left(h_{2}\right.$ is $\left.h_{\mathrm{SM}}\right)$ case and for about $96 \%$ of the surviving samples in the light- $a_{1}\left(h_{1}\right.$ is $h_{\mathrm{SM}}$ ) case (for the remaining $4 \%$ of the surviving samples in the light- $a_{1}$ case, the decay $h_{\mathrm{SM}} \rightarrow a_{1} a_{1}$ is usually kinematically forbidden so that the ratio of $h_{\mathrm{SM}} \rightarrow b \bar{b}$ may exceed $60 \%$ ). Another interesting feature shown in Figure 7 is that, due to the open-up of the exotic decays, $h_{\mathrm{SM}}$ may be significantly lighter than the LEP bound. This situation is favored by the fit of the precision electroweak data and is of great theoretical interest [81].

Since the conventional decay modes of $h_{\mathrm{SM}}$ may be greatly suppressed, especially in the light- $h_{1}$ case which can give a rather large $\tilde{x}$-nucleon scattering rate, the LHC search for $h_{\mathrm{SM}}$ via the traditional channels may become difficult. Now the LHC observed a new particle in the mass region around $125-126 \mathrm{GeV}$ which is the most probable long sought Higgs boson [82]. In this mass range, the most important discovering channel of $h_{\mathrm{SM}}$ at the LHC is the di-photon signal. In Figure 8 we give the ratio of the di-photon production rate to the SM at the LHC with $\sqrt{s}=7 \mathrm{TeV}$. In calculating the rate, we used the narrow width approximation and only considered the leading contributions to $p p \rightarrow h_{\mathrm{SM}}$ from top quark, bottom quark, and the squark loops.

Figure 8 indicates that, compared with the SM prediction, the ratio in the NMSSM in the light $\tilde{X}_{1}^{0}$ scenario is suppressed to be less than 0.4 for the light- $h_{1}$ case. For the light- $a_{1}$ case, most samples (about 96\%) predict the same conclusion. Since in the light- $h_{1}$ case the 

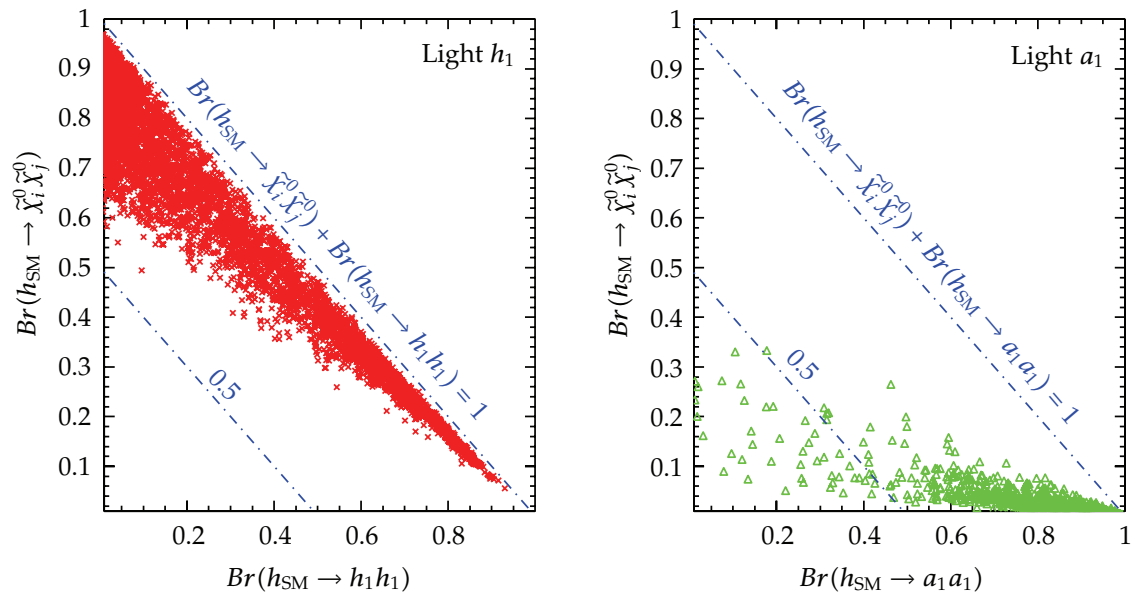

(a)
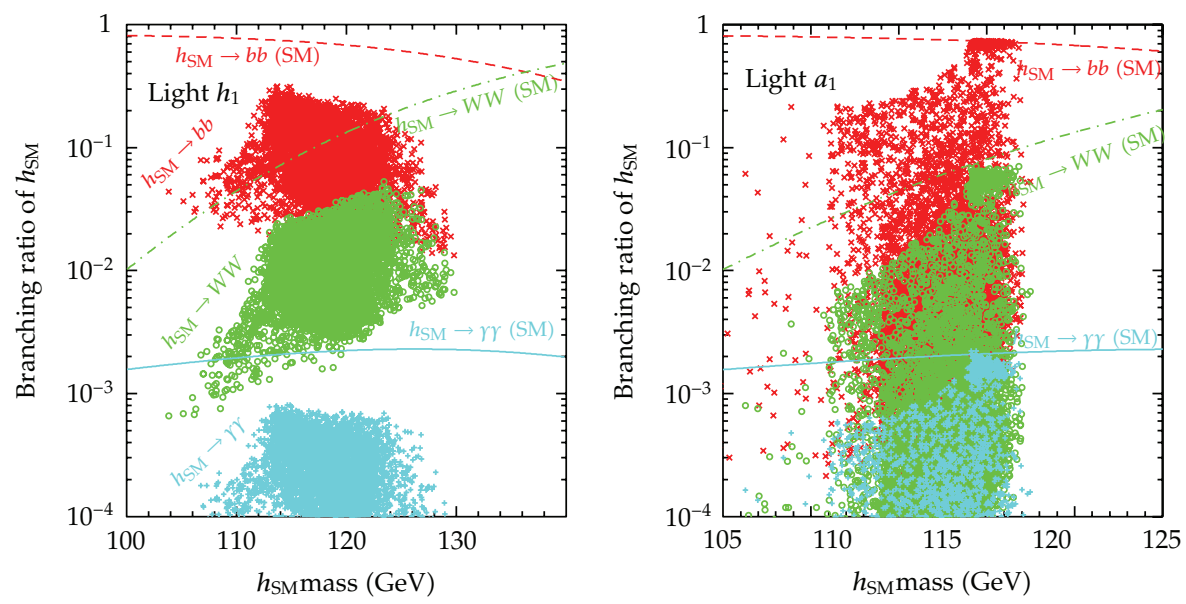

(b)

Figure 7: Same as Figure 6, but showing the decay branching ratios of the SM-like Higgs boson $h_{\mathrm{SM}}$. Here $\mathrm{Br}\left(h_{\mathrm{SM}} \rightarrow \tilde{X}_{i}^{0} \tilde{X}_{j}^{0}\right)$ denotes the total rates for all possible $h_{\mathrm{SM}} \rightarrow \tilde{X}_{i}^{0} \tilde{X}_{j}^{0}$ decays (taken for [47]).

$\tilde{X}$-nucleon scattering rate can reach the CoGeNT sensitivity, this means that in the framework of the NMSSM the CoGeNT search for the light dark matter will be correlated with the LHC search for the Higgs boson via the di-photon channel. We checked that once the future XENON experiment fails in observing dark matter, less than $1 \%$ of the surviving samples in light $a_{1}$ case predict the ratio of di-photon signal larger than 0.4 .

\section{General Extension for the Explanation to PAMELA}

To explain the PAMELA excess by dark matter annihilation, there are some challenges. First, dark matter must annihilate dominantly into leptons since PAMELA has observed no excess of antiprotons [43, 44] (however, as pointed in [83], this statement may be not so solid due to the significant astrophysical uncertainties associated with their propagation). Second, the 


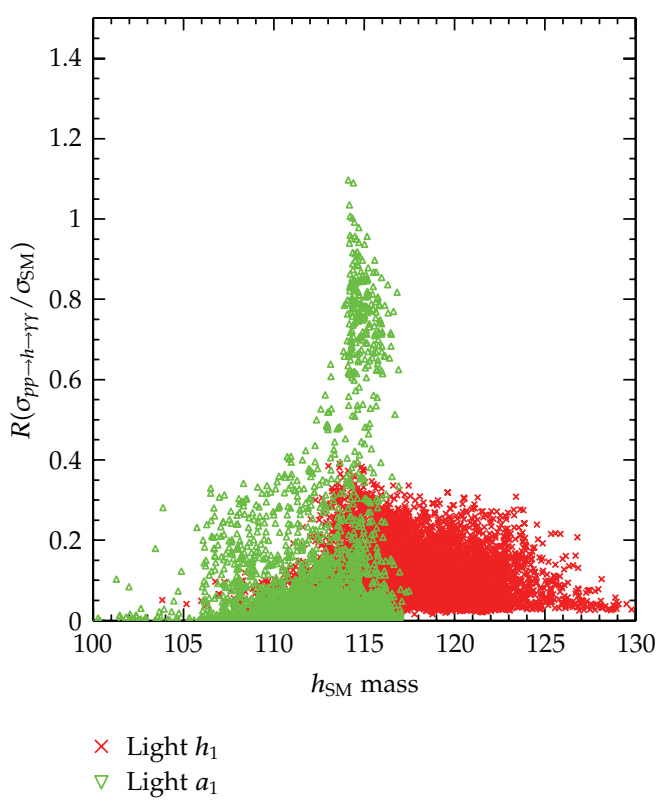

Figure 8: Same as Figure 6, but showing the diphoton production rate of the SM-like Higgs boson at the LHC.

explanation of PAMELA excess requires an annihilation rate which is too large to explain the relic abundance if dark matter is produced thermally in the early universe. To tackle these difficulties, a new theory of dark matter was proposed in [84]. In this new theory the Sommerfeld effect of a new force in the dark sector can greatly enhance the annihilation rate when the velocity of dark matter is much smaller than the velocity at freeze-out in the early universe, and dark matter annihilates into light particles which are kinematically allowed to decay to muons or electrons.

The above fancy idea is hard to realize in the MSSM, because there is not a new force in the neutralino dark matter sector to induce the Sommerfeld enhancement, and neutralino dark matter annihilates largely to final states consisting of heavy quarks or gauge and/or Higgs bosons [52, 85-88]. However, as discussed in [89], in a general extension of the MSSM by introducing a singlet Higgs superfield, the idea in [84] can be realized by the singlinolike neutralino dark matter.

(i) The singlino dark matter annihilates to the light singlet Higgs bosons, and the relic density can be naturally obtained from the interaction between singlino and singlet Higgs bosons.

(ii) The singlet Higgs bosons, not related to electroweak symmetry breaking, can be light enough to be kinematically allowed to decay dominantly into muons or electrons through the tiny mixing with the Higgs doublets.

(iii) The Sommerfeld enhancement needed in dark matter annihilation for the explanation of PAMELA result can be induced by the light singlet Higgs boson.

In the following section, we will show how does this happen: the Higgs decay is also investigated. 


\subsection{Higgs and Neutralinos Spectrum}

If we introduce a singlet Higgs to the MSSM in general, the renormalizable holomorphic superpotential of Higgs is given by [89]

$$
W=\mu \widehat{H}_{u} \cdot \widehat{H}_{d}+\lambda \widehat{S} \widehat{H}_{u} \cdot \widehat{H}_{d}+\eta \widehat{S}+\frac{1}{2} \mu_{s} \widehat{S}^{2}+\frac{1}{3} \kappa \widehat{S}^{3}
$$

which include linear term, quadratic term, cubic term of singlet superfield (like Wess-Zumino model [90]). Note that, in such case, we do not require the singlet to solve the $\mu$ problem. The soft SUSY-breaking terms are given by

$$
\begin{aligned}
V_{\text {soft }}= & \tilde{m}_{u}^{2}\left|H_{u}\right|^{2}+\tilde{m}_{d}^{2}\left|H_{d}\right|^{2}+\tilde{m}_{s}^{2}|S|^{2} \\
& +\left(B \mu H_{u} \cdot H_{d}+\lambda A_{\lambda} H_{u} \cdot H_{d} S+C \eta S+\frac{1}{2} B_{s} \mu_{S} S^{2}+\frac{1}{3} \kappa A_{\kappa} S^{3}+\text { h.c. }\right) .
\end{aligned}
$$

After the Higgs fields develop the VEVs $v_{u}, v_{d}$, and $s$, that is, we get the similar Higgs spectrum as the NMSSM and the nMSSM which is the following.

(1) The CP-even Higgs mass matrix in the basis $\left(\phi_{u}, \phi_{d}, \sigma\right)$ is given by

$$
\begin{aligned}
& \mathcal{M}_{h, 11}=g^{2} v_{u}^{2}+\cot \beta\left[\lambda s\left(A_{\curlywedge}+\kappa s+\mu_{s}\right)+B \mu\right], \\
& \mathcal{M}_{h, 22}=g^{2} v_{d}^{2}+\tan \beta\left[\lambda s\left(A_{\curlywedge}+\kappa s+\mu_{s}\right)+B \mu\right], \\
& \mathcal{M}_{h, 33}=\lambda\left(A_{\curlywedge}+\mu_{s}\right) \frac{v_{u} v_{d}}{s}-\lambda \frac{\mu}{s}\left(v_{u}^{2}+v_{d}^{2}\right)+\kappa s\left(A_{\kappa}+4 \kappa s+3 \mu_{s}\right)-\frac{C \eta}{s}, \\
& \mathcal{M}_{h, 12}=\left(2 \lambda^{2}-g^{2}\right) v_{u} v_{d}-\lambda s\left(A_{\curlywedge}+\kappa s+\mu_{s}\right)-B \mu, \\
& \mathcal{M}_{h, 13}=2 \lambda(\mu+\lambda s) v_{u}-\lambda v_{d}\left(A_{\curlywedge}+2 \kappa s+\mu_{s}\right), \\
& \mathcal{M}_{h, 23}=2 \lambda(\mu+\lambda s) v_{d}-\lambda v_{u}\left(A_{\curlywedge}+2 \kappa s+\mu_{s}\right),
\end{aligned}
$$

where $g^{2}=\left(g_{1}^{2}+g_{2}^{2}\right) / 2$ with $g_{1}$ and $g_{2}$ being, respectively, the coupling constant of $S U(2)$ and $U(1)$ in the SM.

(2) The CP-odd Higgs mass matrix $\boldsymbol{M}_{a}$ is given by

$$
\begin{gathered}
\mathcal{M}_{a, 11}=(\tan \beta+\cot \beta)\left[\lambda s\left(A_{\curlywedge}+\kappa s+\mu_{s}\right)+B \mu\right] \\
\mathcal{M}_{a, 22}=4 \lambda \kappa v_{u} v_{d}+\lambda\left(A_{\curlywedge}+\mu_{s}\right) \frac{v_{u} v_{d}}{s}-\lambda \frac{\mu}{s}\left(v_{u}^{2}+v_{d}^{2}\right) \\
-\kappa s\left(3 A_{\kappa}+\mu_{s}\right)-\frac{C \eta}{s}-2 B_{s} \mu_{s} \\
\boldsymbol{M}_{a, 12}=\lambda \sqrt{v_{u}^{2}+v_{d}^{2}}\left(A_{\curlywedge}-2 \kappa s-\mu_{s}\right) .
\end{gathered}
$$


Note that here we have dropped the Goldstone mode; thus, there left a $2 \times 2$ mass matrix in the basis $(\tilde{A}, \xi)$, and it can be diagonalized by an orthogonal $2 \times 2$ matrix $P^{\prime}$ and the physical CP-odd states $a_{i}$ are given by (ordered as $m_{a_{1}}<m_{a_{2}}$ )

$$
\begin{aligned}
& a_{1}=P_{11}^{\prime} \tilde{A}+P_{12}^{\prime} S_{I}=P_{11}^{\prime}\left(\cos \beta \varphi_{u}+\sin \beta \varphi_{d}\right)+P_{12}^{\prime} \xi, \\
& a_{2}=P_{21}^{\prime} \tilde{A}+P_{22}^{\prime} S_{I}=P_{21}^{\prime}\left(\cos \beta \varphi_{u}+\sin \beta \varphi_{d}\right)+P_{22}^{\prime} \xi .
\end{aligned}
$$

(3) The charged Higgs mass matrix $\mathcal{M}_{ \pm}$in the basis $\left(H_{u}^{+}, H_{d}^{+}\right)$is given by

$$
\mathcal{M}_{ \pm}=\left(\lambda s\left(A_{\curlywedge}+\kappa s+\mu_{s}\right)+B \mu+h_{u} h_{d}\left(\frac{g_{2}^{2}}{2}-\lambda^{2}\right)\right)\left(\begin{array}{cc}
\cot \beta & 1 \\
1 & \tan \beta
\end{array}\right) .
$$

(4) The neutralino mass matrix is

$$
\mathcal{M}_{0}=\left(\begin{array}{ccccc}
M_{1} & 0 & m_{Z} s_{W} s_{b} & -m_{Z} s_{W} c_{b} & 0 \\
0 & M_{2} & -m_{Z} c_{W} s_{b} & m_{Z} c_{W} c_{b} & 0 \\
m_{Z} s_{W} s_{b} & -m_{Z} s_{W} s_{b} & 0 & -\mu & -\lambda v c_{b} \\
-m_{Z} s_{W} c_{b} & -m_{Z} c_{W} c_{b} & -\mu & 0 & -\lambda v s_{b} \\
0 & 0 & -\lambda v c_{b} & -\lambda v s_{b} & 2 \kappa s+\mu_{s}
\end{array}\right) .
$$

\subsection{Explanation of PAMELA and Implication on Higgs Decays}

To explain the observation of PAMELA, $a_{1}$ is singlet dominant, while $h_{1}$ is singlet-dominant, and the next-to-lightest $h_{2}$ is doublet-dominant $\left(h_{\mathrm{SM}}\right)$. We use the notation:

$$
a \equiv a_{1}, \quad h \equiv h_{1}, \quad h_{\mathrm{SM}} \equiv h_{2} .
$$

As discussed in [89], when the lightest neutralino $\widetilde{X}_{1}^{0}$ in (2.8) is singlino-dominant, it can be a perfect candidate for dark matter. As shown in Figure 9, such singlino dark matter annihilates to a pair of light singlet Higgs bosons followed by the decay $h \rightarrow a a$ ( $h$ has very small mixing with the Higgs doublets and thus has very small couplings to the SM fermions). In order to decay dominantly into muons, $a$ must be light enough. Further, in order to induce the Sommerfeld enhancement, $h$ must also be light enough. From the superpotential term $\kappa \widehat{S}^{3}$ we know that the couplings $h \widetilde{\chi}_{1}^{0} \widetilde{X}_{1}^{0}$ and $a \widetilde{\chi}_{1}^{0} \widetilde{X}_{1}^{0}$ are proportional to $\kappa$. To obtain the relic density of dark matter, $\kappa$ should be $\mathcal{O}(1) . h, a$ are singlet-dominant, and $\tilde{\chi}_{1}^{0}$ is singlino-dominant, this implies small mixing between singlet and doublet Higgs fields. From the superpotential in (4.1) we see that this means that the mixing parameter $\lambda$ must be small enough. On the other 


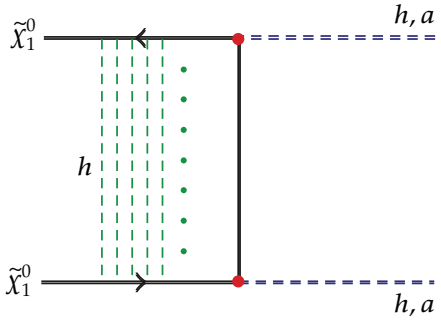

(a)

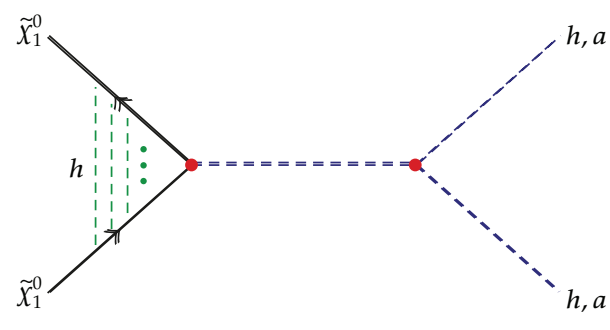

(b)

Figure 9: Feynman diagrams for singlino dark matter annihilation where Sommerfeld enhancement is induced by exchanging $h$ (taken from [45]).

hand, from (4.5) and (4.10) lightness of $h_{1}$ and $a_{1}$ also requires $\lambda$ and other term approaching to zero. Therefore, in our scan we require that parameters $A_{\kappa}$ and $B_{s}$ have the relation:

$$
\begin{aligned}
A_{\kappa} & \sim\left(-4 \kappa s-3 \mu_{s}+\frac{C \eta}{\kappa s^{2}}\right), \\
2 B_{S} \mu_{s} & \sim\left(-3 A_{\kappa} \kappa s-\mu_{s} \kappa s-\frac{C \eta}{s}\right),
\end{aligned}
$$

to realize light $h_{1}$ and $a_{1}$.

The numerical results of this model are displayed in different planes in Figures 10-12. We see from Figure 10 that, in the range $2 m_{\mu}<m_{a}<2 m_{\pi}$, $a$ decays dominantly into muons. It is clear that $h$ can be as light as a few $\mathrm{GeV}$, which is light enough to induce the necessary Sommerfeld enhancement as shown in Figure 11.

In left plot of Figure 12, we show the branching ratios of $h_{\mathrm{SM}}$ decays. We see that in the allowed parameter space $h_{\mathrm{SM}}$ tends to decay into $a a$ or $h h$ instead of $b \bar{b}$. This can be understood as follows: the MSSM parameter space is stringently constrained by the LEP experiments if $h_{\mathrm{SM}}$ is relatively light and decays dominantly to $b \bar{b}$, and to escape such stringent constraints $h_{\mathrm{SM}}$ tends to have exotic decays into $a a$ or $h h$. As a result, the allowed parameter space tends to favor a large $A_{\kappa}$, as shown in right plot of Figure 12, which greatly enhances the couplings $h_{\mathrm{SM}} a a$ and $h_{\mathrm{SM}} h h$ through the soft term $\kappa A_{\kappa} S^{3}$ although $S$ has a small mixing with the doublet Higgs bosons. Such an enhancement can be easily seen. Take the coupling $h_{\mathrm{SM}} h h$ as an example, the soft term $\kappa A_{\kappa} S^{3}$ gives a term $\kappa A_{\kappa} \sigma^{3}$ which then gives the interaction $\kappa A_{\kappa}\left(U_{13}^{H}\right)^{2} U_{23}^{H} h_{\mathrm{SM}} h h$ because $\sigma=U_{13}^{H} h_{1}+U_{23}^{H} h_{2}+U_{33}^{H} h_{3}$ with $h_{1} \equiv h$ and $h_{2} \equiv h_{\mathrm{SM}}$ (see (2.6) and (4.15)). Although the mixing $\left(U_{13}^{H}\right)^{2} U_{23}^{H}$ is small for a small $\lambda$, a large $A_{\kappa}$ can enhance the coupling $h_{\mathrm{SM}} h h$. Note that as the mass of the observed Higgs boson at the LHC is around $125 \mathrm{GeV}$, thus in the MSSM, the dominant decay mode of $h_{\mathrm{SM}}$ is $b \bar{b}$. In this general singlet extension of the MSSM, its dominant decay mode may be changed to aa or $h h$, as shown in our above results.

Finally, we note that, for the specified singlet extensions like the nMSSM and the NMSSM, the explanation of PAMELA and relic density through Sommerfeld enhancement is not possible. The reason is that the parameter space of such models is stringently constrained by various experiments and dark matter relic density as shown in the above section, and, 


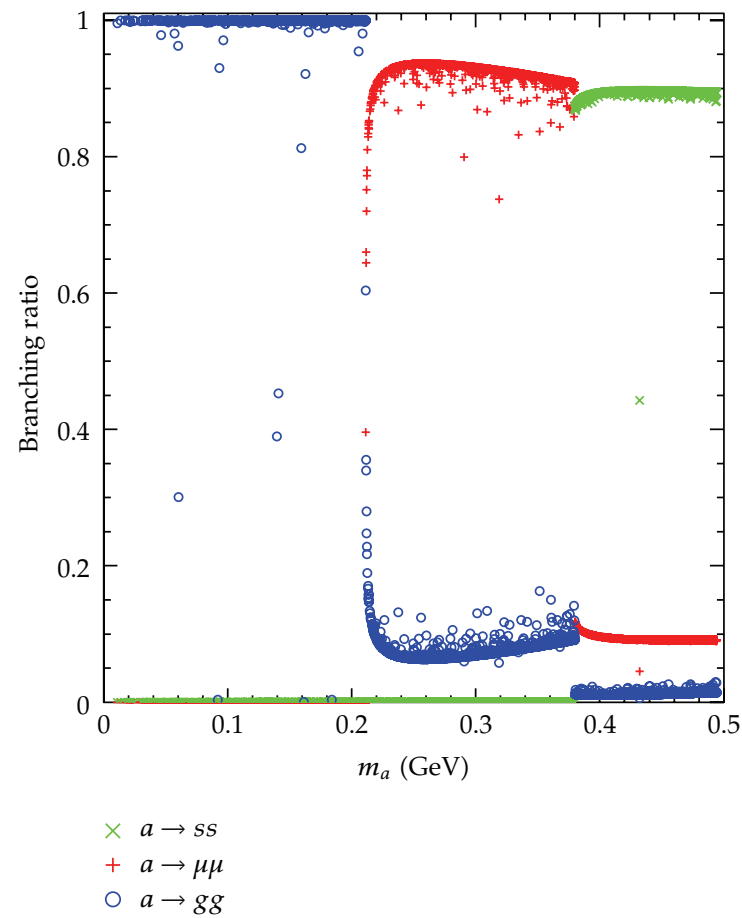

Figure 10: The scatter plots showing the decay branching ratios $a \rightarrow \mu^{+} \mu^{-}$(muon), $a \rightarrow g g$ (gluon), and $a \rightarrow s \bar{s}$ ( $s$-quark) versus $m_{a}$ for $\lambda=10^{-3}$ (taken from [45]).

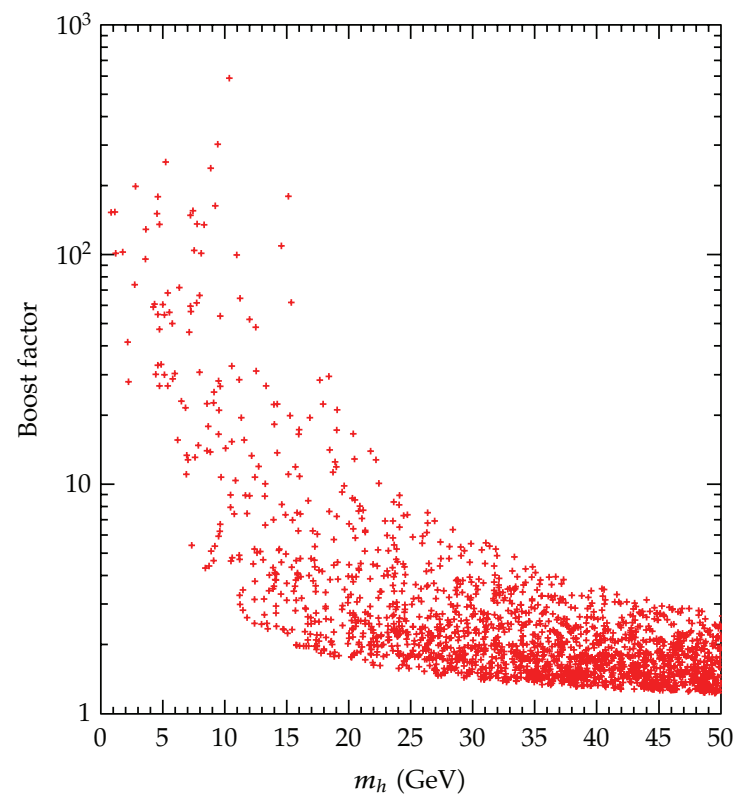

Figure 11: Same as Figure 10, but showing the Sommerfeld enhancement factor induced by $h$ (taken from [45]). 


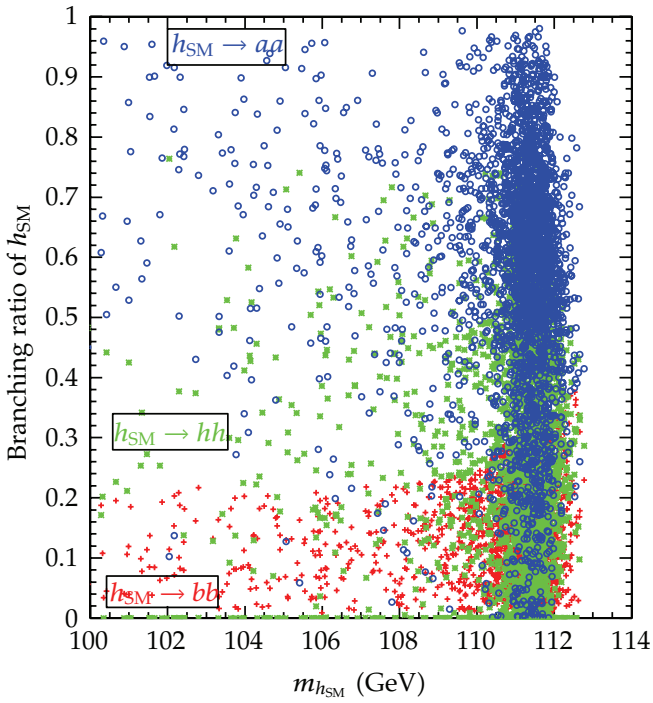

(a)

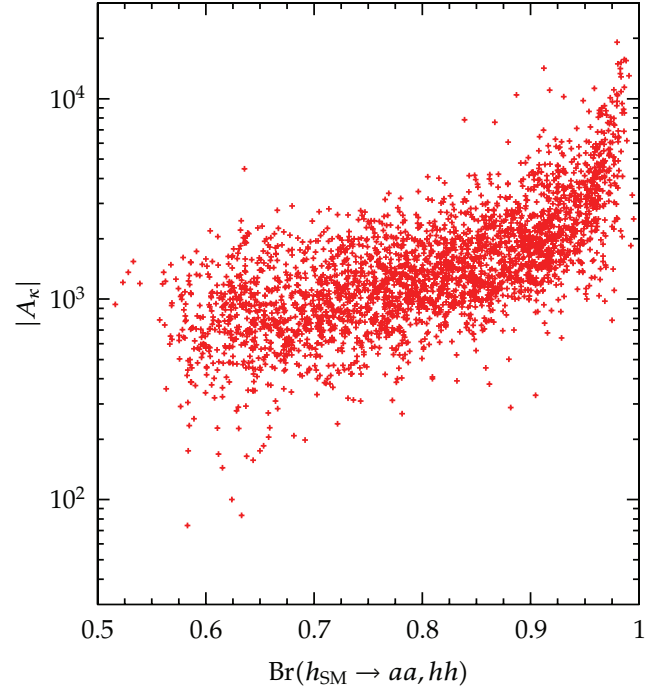

(b)

Figure 12: Same as Figure 10, but showing branching ratio of $h_{\mathrm{SM}} \rightarrow a a, h h$ versus $m_{h_{\mathrm{SM}}}$ and $\left|A_{\kappa}\right|$ versus the branching ratio of $h_{\mathrm{SM}} \rightarrow a a, h h$ (taken from [45]).

as a result, the neutralino dark matter may explain either the relic density or PAMELA, but impossible to explain both via Sommerfeld enhancement. For example, in the nMSSM various experiments and dark matter relic density constrain the neutralino dark matter particle in a narrow mass range [91], which is too light to explain PAMELA.

\section{Summary}

At last we summarize here the SUSY dark matter, and Higgs physics will be changed if we introduce a singlet to the MSSM. Under the latest results of dark matter detection, we have the following.

(1) In the MSSM, the NMSSM, and the NMSSM, the latest detection result can exclude a large part of the parameter space allowed by current collider constraints, and the future SuperCDMS and XENON can cover most of the allowed parameter space.

(2) Under the new dark matter constraints, the singlet sector will decouple from the MSSM-like sector in the NMSSM; thus, the phenomenologies of dark matter and Higgs are similar to the MSSM. The singlet sector makes the nMSSM quite different from the MSSM, the LSP in the nMSSM is singlet dominant, and the SM-like Higgs will mainly decay into the singlet sector. Future precision measurements will give us an opportunity to determine whether the new scalar is from standard model or from SUSY. Perhaps the nMSSM will be the first model excluded for its much larger branching ratio of invisible Higgs decay.

(3) The NMSSM can allow light dark matter at several GeV to exist. Light CP-even or $\mathrm{CP}$-odd Higgs boson must be present so as to satisfy the measured dark matter relic density. In case of the presence of a light CP-even Higgs boson, the light neutralino dark matter can explain the CoGeNT and DAMA/LIBRA results. Further, we find 
that in such a scenario the SM-like Higgs boson will decay predominantly into a pair of light Higgs bosons or a pair of neutralinos, and the conventional decay modes will be greatly suppressed.

(4) The general singlet extension of the MSSM gives a perfect explanation for both the relic density, and the PAMELA result through the Sommerfeld enhanced annihilation into singlet Higgs bosons ( $a$ or $h$ followed by $h \rightarrow a a$ ) with $a$ being light enough to decay dominantly to muons or electrons. Although the light singlet Higgs bosons have small mixing with the Higgs doublets in the allowed parameter space, their couplings with the SM-like Higgs boson $h_{\mathrm{SM}}$ can be enhanced by the soft parameter $A_{\kappa}$. In order to meet the stringent LEP constraints, the $h_{\mathrm{SM}}$ tends to decay into the singlet Higgs pairs $a a$ or $h h$ instead of $b \bar{b}$.

\section{Acknowledgment}

This work was supported in part by the NSFC no. 11005006, no. 11172008, and Doctor Foundation of BJUT no. X0006015201102.

\section{References}

[1] H. E. Haber and G. L. Kane, "The search for supersymmetry: Probing physics beyond the standard model," Physics Reports, vol. 117, no. 2-4, pp. 75-263, 1985.

[2] S. Abel, E. Accomando, G. Anderson et al., "Report of SUGRA Working Group for Run II of the Tevatron," http://arxiv.org/abs/hep-ph/0003154.

[3] LEPSUSYWG, ALEPH, DELPHI, L3 and OPAL Collaborations, LEPSUSYWG/01-03.1 http://lepsusy .web.cern.ch/lepsusy/.

[4] J. Ellis, J. F. Gunion, H. E. Haber, L. Roszkowski, and F. Zwirner, "Higgs bosons in anonminimal supersymmetric model," Physical Review D, vol. 39, no. 3, pp. 844-869, 1989.

[5] M. Drees, "Supersymmetric models with extended higgs sector," International Journal of Modern Physics $A$, vol. 4, no. 14, p. 3635, 1989.

[6] P. N. Pandita, "One-loop radiative corrections to the lightest Higgs scalar mass in the non-minimal supersymmetric standard model," Physics Letters B, vol. 318, no. 2, pp. 338-346, 1993.

[7] P. N. Pandita, "Approximate formulas for the neutralino masses in the nonminimal supersymmetric standard model," Physical Review D, vol. 50, pp. 571-577, 1994.

[8] S. F. King and P. L. White, "Resolving the constrained minimal and next-to-minimal supersymmetric standard models," Physical Review D, vol. 52, pp. 4183-4216, 1995.

[9] B. Ananthanarayan and P. N. Pandita, "The non-minimal supersymmetric standard model with tan $\beta \simeq \mathrm{m}_{\mathrm{t}} / \mathrm{m}_{\mathrm{b}}$," vol. 353, no. 1, pp. 70-78, 1995 .

[10] B. Ananthanarayan and P. N. Pandita, "Particle spectrum in the non-minimal supersymmetric standard model with $\tan \beta \simeq \mathrm{m}_{\mathrm{t}} / \mathrm{m}_{\mathrm{b}}$," Physics Letters B, vol. 371, no. 3-4, pp. 245-251, 1996.

[11] B. Ananthanarayan and P. N. Pandita, "The Nonminimal Supersymmetric Standard Model at Large $\tan \beta, "$ International Journal of Modern Physics A, vol. 12, no. 13, p. 2321, 1997.

[12] B. A. Dobrescu and K. T. Matchev, "Light axion within the next-to-minimal supersymmetric standard model," Journal of High Energy Physics, vol. 2000, no. 09, p. 031, 2000.

[13] V. Barger, P. Langacker, H. S. Lee, and G. Shaughnessy, "Higgs sector in extensions of the minimal supersymmetric standard model," Physical Review D, vol. 73, no. 11, Article ID 115010, 31 pages, 2006.

[14] R. Dermisek and J. F. Gunion, "Escaping the large fine-tuning and little hierarchy problems in the next to minimal supersymmetric model and $\mathrm{h} \rightarrow$ aa decays," Physical Review Letters, vol. 95, no. 4, Article ID 041801, 4 pages, 2005.

[15] G. Hiller, " $b$-physics signals of the lightest $C P$-odd Higgs boson in the next-to-minimal supersymmetric standard model at large tan $\beta$," Physical Review D, vol. 70, no. 3, Article ID 034018, 4 pages, 2004.

[16] F. Domingo and U. Ellwanger, "Updated constraints from B physics on the MSSM and the NMSSM," Journal of High Energy Physics, vol. 2007, no. 12, p. 090, 2007. 
[17] Z. Heng et al., "B meson dileptonic decays in the next-to-minimal supersymmetric model with a light CP-odd Higgs boson," Physical Review D, vol. 77, no. 9, Article ID 095012, 12 pages, 2008.

[18] R. N. Hodgkinson and A. Pilaftsis, "Radiative Yukawa couplings for supersymmetric Higgs singlets at large tan $\beta, "$ Physical Review D, vol. 76, no. 1, Article ID 015007, 12 pages, 2007.

[19] R. N. Hodgkinson and A. Pilaftsis, "Supersymmetric Higgs singlet effects on B-meson flavorchanging neutral current observables at large $\tan \beta, "$ Physical Review D - Particles, Fields, Gravitation and Cosmology, vol. 78, no. 7, Article ID Article number075004, 2008.

[20] W. Wang, Z. Xiong, and J. M. Yang, "Residual effects of heavy sparticles in the bottom quark Yukawa coupling: a comparative study for the MSSM and NMSSM," Physics Letters B, vol. 680, no. 2, pp. 167-171, 2009.

[21] J. Cao and J. M. Yang, "Anomaly of Z $\bar{b}$ coupling revisited in MSSM and NMSSM," Journal of High Energy Physics, vol. 2008, no. 12, p. 006, 2008.

[22] J. Cao and J. M. Yang, "Current experimental constraints on the next-to-minimal supersymmetric standard model with large $\lambda$," Physical Review D, vol. 78, no. 11, Article ID 115001, 8 pages, 2008.

[23] U. Ellwanger, C. Hugonie, and A. M. Teixeira, "The next-to-minimal supersymmetric standard model," Physics Reports, vol. 496, no. 1-2, pp. 1-77, 2010.

[24] J. Cao, Z. Heng, and J. M. Yang, "Rare Z-decay into light $C P$-odd Higgs bosons: a comparative study in different new physics models," Journal of High Energy Physics , vol. 2010, no. 11, p. 110, 2010.

[25] M. Maniatis, "The next-to-minimal supersymmetric extension of the standard model reviewed," International Journal of Modern Physics A, vol. 25, no. 18-19, p. 3505, 2010.

[26] U. Ellwanger, "Higgs bosons in the next-to-minimal supersymmetric standard model at the LHC," The European Physical Journal C, vol. 71, no. 10, p. 1782, 2011.

[27] J. Cao, Z. Heng, J. M. Yang, and J. Zhu, "Higgs decay to dark matter in low energy SUSY: is it detectable at the LHC?" Journal of High Energy Physics, vol. 2012, no. 06, p. 145, 2012.

[28] P. Fayet, Nuclear Physics B, vol. 90, pp. 104-124, 1975.

[29] C. Panagiotakopoulos and K. Tamvakis, "Stabilized NMSSM without domain walls," Physics Letters B, vol. 446, no. 3-4, pp. 224-227, 1999.

[30] C. Panagiotakopoulos and K. Tamvakis, "New minimal extension of MSSM," Physics Letters B, vol. 469, no. 1-4, pp. 145-148, 1999.

[31] C. Panagiotakopoulos and A. Pilaftsis, "Higgs scalars in the minimal nonminimal supersymmetric standard model," Physical Review D, vol. 63, Article ID 055003, 33 pages, 2001.

[32] A. Dedes et al., "Phenomenology of a new minimal supersymmetric extension of the standard model," Physical Review D, vol. 63, no. 5, Article ID 055009, 9 pages, 2001.

[33] A. Menon, D. E. Morrissey, and C. E. M. Wagner, "Electroweak baryogenesis and dark matter in a minimal extension of the MSSM," Physical Review D, vol. 70, no. 3, Article ID 035005, 20 pages, 2004.

[34] V. Barger, P. Langackerb, and H.-S. Leea, "Lightest neutralino in extensions of the MSSM," Physics Letters B, vol. 630, no. 3-4, pp. 85-99, 2005.

[35] C. Balazs, M. Carena, A. Freitas et al., "Phenomenology of the nMSSM from colliders to cosmology," Journal of High Energy Physics, vol. 2007, no. 06, p. 066, 2007.

[36] J. Cao, Z. Heng, and J. M. Yang, "Rare Z-decay into light CP-odd Higgs bosons: a comparative study in different new physics models," Journal of High Energy Physics, vol. 2010, no. 11, p. 110, 2010.

[37] J. Cao, H. E. Logan, and J. M. Yang, "Experimental constraints on the nearly minimal supersymmetric standard model and implications for its phenomenology," Physical Review D, vol. 79, no. 9, Article ID 091701, 5 pages, 2009.

[38] Z. Ahmed, D. S. Akerib, S. Arrenberg et al., "Dark matter search results from the CDMS II experiment," Science, vol. 327, no. 5973, pp. 1619-1621, 2010.

[39] E. Aprile, K. Arisaka, F. Arneodo et al., "First dark matter results from the XENON100 experiment," Physical Review Letters, vol. 105, no. 13, Article ID 131302, 5 pages, 2010.

[40] E. Aprile et al., "Dark matter results from 100 live days of XENON100 data," Physical Review Letters, vol. 107, no. 13, Article ID 131302, 6 pages, 2011.

[41] C. E. Aalseth, P. S. Barbeau, N. S. Bowden et al., "Results from a search for light-mass dark matter with a $p$-type point contact germanium detector," Physcal Review Letters, vol. 106, no. 13, Article ID 131301, 4 pages, 2011.

[42] D. Hooper, J. I. Collar, J. Hall et al., “Consistent dark matter interpretation for CoGeNT and DAMA/ LIBRA," Physical Review D, vol. 82, no. 12, Article ID 123509, 8 pages, 2010.

[43] O. Adriani, G. C. Barbarino, G. A. Bazilevskaya et al., "An anomalous positron abundance in cosmic rays with energies 1.5-100 GeV," Nature, vol. 458, pp. 607-609, 2009. 
[44] O. Adriani, G. A. Bazilevskaya, O. Adriani et al., "New measurement of the antiproton-to-proton flux ratio up to $100 \mathrm{geV}$ in the cosmic radiation," Physical Review Letters, vol. 102, no. 5, Article ID 051101, 5 pages, 2009.

[45] W. Wang, Z. Xiong, J. M. Yang, and L.-X. Yu, “Dark matter in the singlet extension of MSSM: explanation of Pamela and implication on Higgs phenomenology," Journal of High Energy Physics, vol. 2009, no. 11, p. 053, 2009.

[46] J. Cao, K.-I. Hikasa, W. Wang, J. M. Yang, and L.-X. Yu, "SUSY dark matter in light of CDMS II results: a comparative study for different models," Journal of High Energy Physics, vol. 2010, no. 7, p. 44, 2010.

[47] J.-J. Cao, K.-I. Hikasa, W. Wang, and J. M. Yang, "Light dark matter in NMSSM and implication on Higgs phenomenology," Physics Letters B, vol. 703, no. 3, pp. 292-297, 2011.

[48] K. Nakamura and Particle Data Group, "Review of particle physics," Journal of Physics G, vol. 37, no. 7A, Article ID 075021, 2010.

[49] P. Draper, T. Liu, C. E. M. Wagner et al., "Dark light-Higgs bosons,” Physical Review Letters, vol. 106, Article ID 121805, 4 pages, 2011.

[50] U. Ellwanger, J. F. Gunion, and C. Hugonie, "NMHDECAY: a fortran code for the Higgs masses, couplings and decay widths in the NMSSM," Journal of High Energy Physics, vol. 2005, no. 02, p. 066, 2005.

[51] M. Drees and M. M. Nojiri, "Neutralino-nucleon scattering reexamined," Physical Review D, vol. 48, no. 8, pp. 3483-3501, 1993.

[52] G. Junman, M. Kamionkowski, and K. Griest, "Supersymmetric dark matter," Physics Reports, vol. 267, pp. 5-6, 1996.

[53] J. R. Ellis, K. A. Olive, and C. Savage, "Hadronic uncertainties in the elastic scattering of supersymmetric dark matter," Physical Review D, vol. 77, no. 6, Article ID 065026, 15 pages, 2008.

[54] A. Bottino, F. Donato, N. Fornengo, and S. Scopel, "Size of the neutralino-nucleon cross-section in the light of a new determination of the pion-nucleon sigma term," Astroparticle Physics, vol. 18, no. 2, pp. 205-211, 2002.

[55] H. Ohki, H. Fukaya, S. Hashimoto et al., "Nucleon sigma term and strange quark content from lattice QCD with exact chiral symmetry," Physical Review D, vol. 78, no. 5, Article ID 054502, 12 pages, 2008.

[56] D. Toussaint and W. Freeman, "Strange quark condensate in the nucleon in 2+1 flavor QCD," Physical Review Letters, vol. 103, no. 12, Article ID 122002, 4 pages, 2009.

[57] J. Giedt, A. W. Thomas, and R. D. Young, "Dark matter constrained minimal supersymmetric standard model, and lattice QCD," Physical Review Letters, vol. 103, no. 20, Article ID 201802, 4 pages, 2009.

[58] J. Cao, K.-I. Hikasa, W. Wang, J. M. Yang, and L.-X. Yu, “Dark matter direct detection constraints on the minimal supersymmetric standard model and implications for LHC Higgs boson searches," Physical Review D, vol. 82, no. 5, Article ID 051701(R), 5 pages, 2010.

[59] J. Cao, W. Wang, and J. M. Yang, "Split-SUSY dark matter in light of direct detection limits," Physics Letters B, vol. 706, no. 1, pp. 72-76, 2011.

[60] H. P. Nilles, "Supersymmetry, supergravity and particle physics," Physics Reports, vol. 110, pp. 1-162, 1984.

[61] D. Feldman, Z. Liu, and P. Nath, "Low mass neutralino dark matter in the minimal supersymmetric standard model with constraints from $B_{s} \rightarrow \mu^{+} \mu^{-}$and Higgs boson search limits," Physical Review D, vol. 81, no. 11, Article ID 117701, 4 pages, 2010.

[62] A. V. Belikov, J. F. Gunion, D. Hooper, and T. M. P. Tait, “CoGeNT, DAMA, and light neutralino dark matter," Physics Letters B, vol. 705, pp. 82-86, 2011.

[63] N. Fornengo, S. Scopel, and A. Bottino, "Discussing direct search of dark matter particles in the minimal supersymmetric extension of the standard model with light neutralinos," Physical Review D, vol. 83, no. 1, Article ID 015001, 22 pages, 2011.

[64] S. Scopel, S. Choi, N. Fornengo, and A. Bottino, “Impact of the recent results by the CMS and ATLAS collaborations at the CERN Large Hadron Collider on an effective minimal supersymmetric extension of the standard model," Physical Review D, vol. 83, no. 9, Article ID 095016, 6 pages, 2011.

[65] R. Gaitskell, V. Mandic, and J. Filippini, http://dmtools.berkeley.edu/limitplots, and, http:// dmtools.brown.edu:8080.

[66] G. Belanger, F. Boudjema, C. Hugonie, A. Pukhov, and A. Semenov, "Relic density of dark matter in the next-to-minimal supersymmetric standard model," Journal of Cosmology and Astroparticle Physics, vol. 2005, no. 09, p. 001, 2005.

[67] H. K. Dreiner, S. Heinemeyer, O. Kittel, U. Langenfeld, A. M. Weber, and G. Weiglein, “Mass bounds on a very light neutralino," European Physical Journal C, vol. 62, no. 3, pp. 547-572, 2009.

[68] L. Calibbi, T. Ota, and Y. Takanishi, "Light Neutralino in the MSSM: a playground for dark matter, flavor physics and collider experiments," Journal of High Energy Physics, vol. 2011, no. 07, p. 013, 2011. 
[69] D. T. Cumberbatch, D. E. Lopez-Fogliani, L. Roszkowski, R. R. de Austri, and Y.-L. S. Tsai, "Is light neutralino as dark matterstill viable?" http://arxiv.org/abs/1107.1604 .

[70] A. Choudhury and A. Datta, "Many faces of low mass neutralino dark matter in the unconstrained MSSM, LHC data and new signals," Journal of High Energy Physics, vol. 2012, no. 06, p. 006, 2012.

[71] D. Feldman, Z. Liu, and P. Nath, "Low mass neutralino dark matter in the minimal supersymmetric standard model with constraints from $B_{s} \rightarrow \mu^{+} \mu^{-}$and Higgs boson search limits," Physical Review D, vol. 81, no. 11, Article ID 117701, 4 pages, 2010.

[72] E. Kuflik, A. Pierce, and K. M. Zurek, "Light neutralinos with large scattering cross sections in the minimal supersymmetric standard model," Physical Review D, vol. 81, no. 11, Article ID 111701, 5 pages, 2010.

[73] J. F. Gunion, A. V. Belikov, and D. Hooper, "CoGeNT, DAMA, and neutralinodark matter in the nextto-minimal supersymmetric standard model," http://arxiv.org/abs/1009.2555 .

[74] D. A. Vasquez, G. Bélanger, C. Bœhm et al., "Can neutralinos in the MSSM and NMSSM scenarios still be light?" Physical Review D, vol. 82, no. 11, Article ID 115027, 11 pages, 2010.

[75] R. Dermisek and J. F. Gunion, "Direct production of a light CP-odd Higgs boson at the Tevatron and LHC," Physical Review D, vol. 81, no. 5, Article ID 055001, 13 pages, 2010.

[76] R. Dermisek and J. F. Gunion, "New constraints on a light CP-odd Higgs boson and related NMSSM ideal Higgs scenarios," Physical Review D, vol. 81, no. 7, Article ID 075003, 16 pages, 2010.

[77] A. V. Belikov, J. F. Gunion, D. Hooper, and T. M. P. Tait, "CoGeNT, DAMA, and light neutralino dark matter," Physical Letters B, vol. 705, no. 1-2, pp. 82-86, 2011.

[78] R. Kappl, M. Ratza, and M. W. Winkler, "Light dark matter in the singlet-extended MSSM," Physics Letters B, vol. 695, no. 1-4, pp. 169-173, 2011.

[79] J. Cao, H. E. Logan, and J. M. Yang, "Experimental constraints on the nearly minimal supersymmetric standard model and implications for its phenomenology," Physical Review D, vol. 79, no. 9, Article ID 091701, 5 pages, 2009.

[80] D. Das and U. Ellwanger, "Light dark matter in the NMSSM: upper bounds on direct detection cross sections," Journal of High Energy Physics, vol. 2010, no. 09, p. 085, 2010.

[81] R. Dermisek and J. F. Gunion, "Escaping the large fine-tuning and little hierarchy problems in the next to minimal supersymmetric model and $h \rightarrow$ aa decays," Physical Review Letters, vol. 95, no. 4, Article ID 041801, 4 pages, 2005.

[82] http://www.interactions.org/cms/?pid=1031893 .

[83] P. Grajek, G. L. Kane, D. J. Phalen, A. Pierce, and S. Watson, "Is the PAMELA positron excess winos?" Physical Review D, vol. 79, no. 4, Article ID 043506, 2009.

[84] N. Arkani-Hamed, D. P. Finkbeiner, T. R. Slatyer, and N. Weiner, "A theory of dark matter," Physical Review D, vol. 79, no. 1, Article ID 015014, 16 pages, 2009.

[85] E. A. Baltz, J. Edsjo, K. Freese, and P. Gondolo, "Cosmic ray positron excess and neutralino dark matter," Physical Review D, vol. 65, no. 6, Article ID 063511, 10 pages, 2002.

[86] G. L. Kane, L. T. Wang, and T. T. Wang, "Supersymmetry and the cosmic ray positron excess," Physics Letters B, vol. 536, no. 3-4, pp. 263-269, 2002.

[87] G. L. Kane, L. T. Wang, and J. D. Wells, "Supersymmetry and the positron excess in cosmic rays," Physical Review D, vol. 65, no. 5, Article ID 057701, 4 pages, 2002.

[88] K. Ishiwata, S. Matsumoto, and T. Moroi, "Cosmic-ray positron from superparticle dark matter and the PAMELA anomaly," Physical Letters B, vol. 675, no. 5, pp. 446-449, 2009.

[89] D. Hooper and T. M. P. Tait, "Neutralinos in an extension of the minimal supersymmetric standard model as the source of the PAMELA positron excess," Physical Review D, vol. 80, no. 5, Article ID 055028, 5 pages, 2009.

[90] J. Wess and J. Bagger, Supersymmetry and Supergravity, Princeton Series in Physics, Princeton University Press, Princeton, NJ, USA, 2nd edition, 1992.

[91] Y. Bai, M. Carena, and J. Lykken, "PAMELA excess from neutralino annihilation in the NMSSM," Physical Review D, vol. 80, no. 5, Article ID 055004, 17 pages, 2009. 

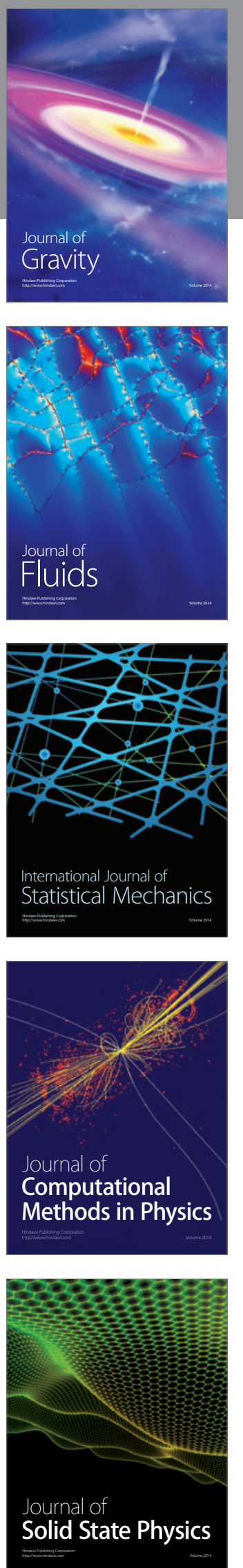

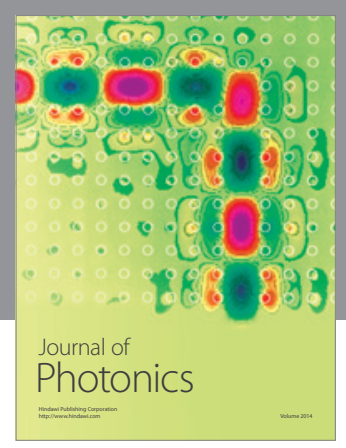

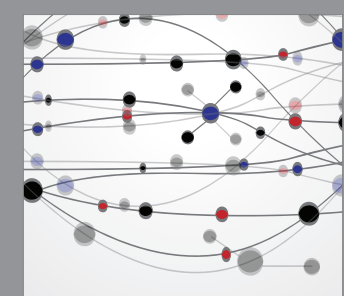

The Scientific World Journal
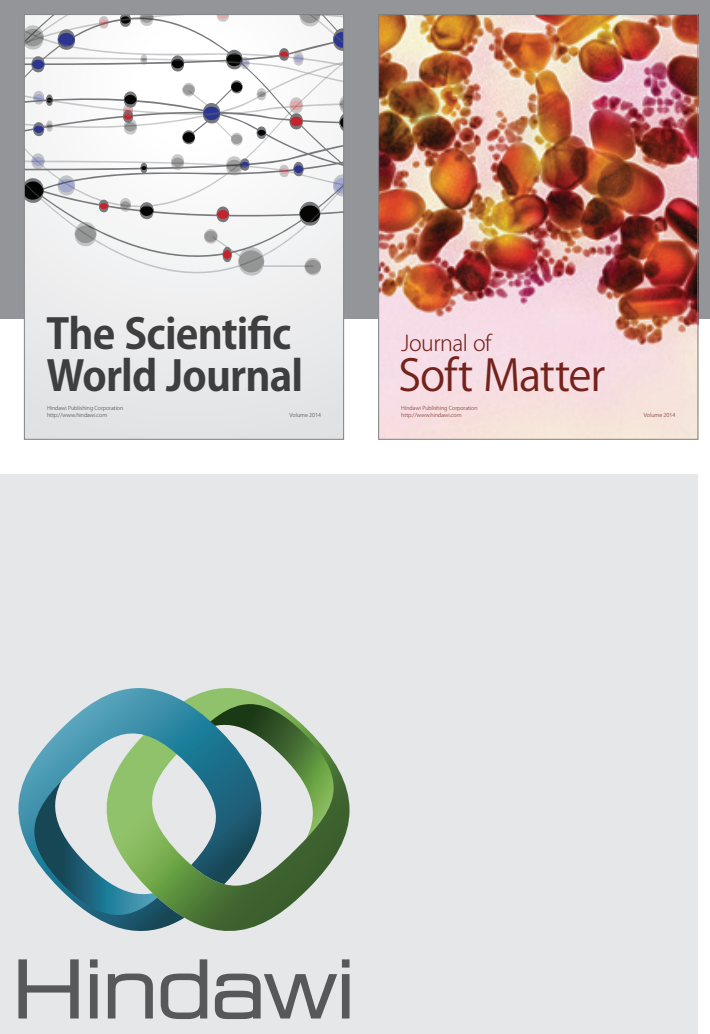

Submit your manuscripts at

http://www.hindawi.com
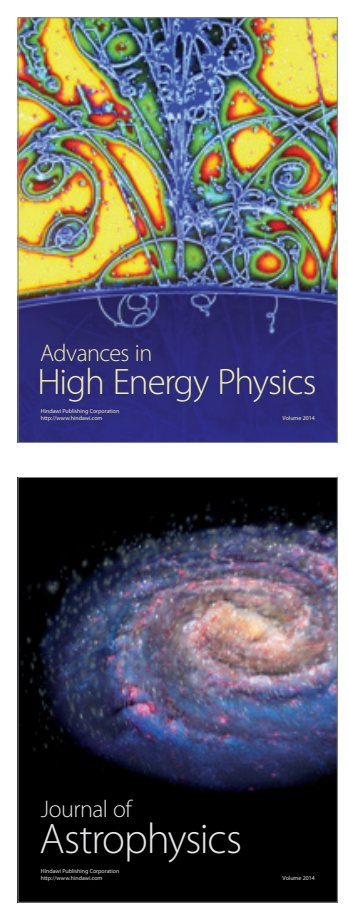
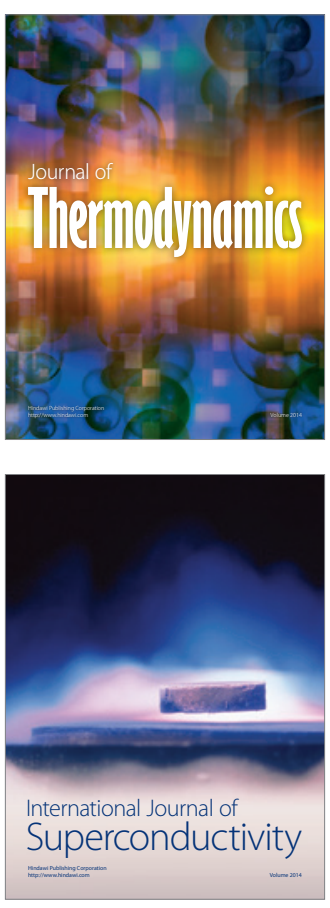
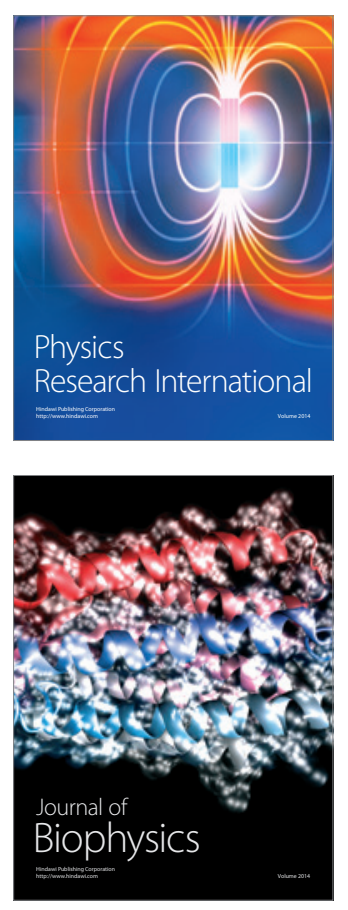
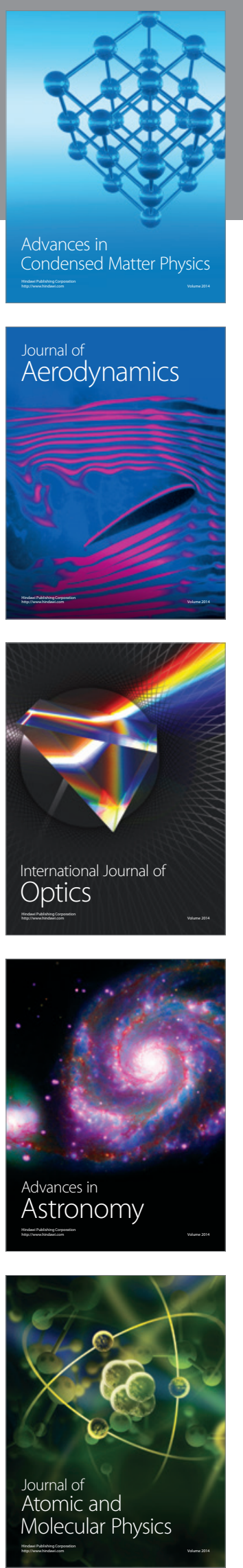